\title{
THE RATE OF CONVERGENCE IN THE METHOD OF ALTERNATING PROJECTIONS
}

\author{
C. BADEA, S. GRIVAUX, AND V. MÜLLER
}

\begin{abstract}
The cosine of the Friedrichs angle between two subspaces is generalized to a parameter associated with several closed subspaces of a Hilbert space. This parameter is employed to analyze the rate of convergence in the von Neumann-Halperin method of cyclic alternating projections. General dichotomy theorems are proved, in the Hilbert or Banach space situation, providing conditions under which the alternative QUC/ASC (quick uniform convergence versus arbitrarily slow convergence) holds. Several meanings for ASC are proposed.
\end{abstract}

\section{$\S 1$. INTRODUCTION}

Throughout the paper $H$ is a complex Hilbert space. For a closed linear subspace $S$ of $H$ we denote by $S^{\perp}$ its orthogonal complement in $H$, and by $P_{S}$ the orthogonal projection of $H$ onto $S$. In this paper $N$ denotes a fixed positive integer greater than or equal to 2 .

1A. The method of alternating projections. It was proved by J. von Neumann 27 , p. 475] that for two closed subspaces $M_{1}$ and $M_{2}$ of $H$ with intersection $M=M_{1} \cap M_{2}$, the following convergence result holds:

$$
\lim _{n \rightarrow \infty}\left\|\left(P_{M_{2}} P_{M_{1}}\right)^{n}(x)-P_{M}(x)\right\|=0 \quad(x \in H) .
$$

Denote $T=P_{M_{2}} P_{M_{1}}$; then von Neumann's result says that the iterates $T^{n}$ of $T$ are strongly convergent to $T^{\infty}=P_{M}$. The method of constructing the iterates of $T$ by alternately projecting onto one subspace and then onto the other is called the method of alternating projections. This algorithm, and its variations, occur in several fields, pure or applied. We refer to [10, Chapter 9] as a source for more information.

A generalization of von Neumann's result to $N$ closed subspaces $M_{1}, \ldots, M_{N}$ with intersection $M=M_{1} \cap M_{2} \cap \cdots \cap M_{N}$ was proved by Halperin [16]: for each $x \in H$ we have

$$
\lim _{n \rightarrow \infty}\left\|\left(P_{M_{N}} \ldots P_{M_{2}} P_{M_{1}}\right)^{n}(x)-P_{M}(x)\right\|=0 .
$$

In the present paper, the algorithm provided by Halperin's result will be called the method of cyclic alternating projections.

A Banach space extension of Halperin's result was proved by Bruck and Reich [9]: if $X$ is a uniformly convex Banach space and $P_{j}, 1 \leq j \leq N$, are $N$ norm one projections in $\mathcal{B}(X)$, then the iterates of $T=P_{N} \ldots P_{2} P_{1}$ are strongly convergent. The strong limit $T^{\infty}$ is a projection of norm one onto the intersection of the ranges of $P_{j}$. The same result is valid [3] if $X$ is uniformly smooth and each projection $P_{j}$ is of norm one. It is also

2010 Mathematics Subject Classification. Primary 47A05, 47A10, 41A35.

Key words and phrases. Friedrichs angle, method of alternating projections, arbitrarily slow convergence.

The first two authors were partially supported by ANR Project Blanc DYNOP. The third author was supported by grant No. 201/09/0473 of GA ČR and IAA100190903 of GA AV. 
true [3] if $X$ is a reflexive (complex) Banach space and each projection $P_{j}$ is Hermitian (that is, with real numerical range). We refer to 3 and the references therein for other Banach space results of this type.

An interesting extension of the method of cyclic alternating projections is the method of random alternating projections. Let $P_{j}, 1 \leq j \leq N$, be $N$ orthogonal projections in $\mathcal{B}(H)$, let $M=\bigcap_{j=1}^{N} \operatorname{Ran}\left(P_{j}\right)$, and let $\left(i_{k}\right)_{k \geq 1}$ be a sequence from $\{1,2, \ldots, N\}$ (random samples). The method of random alternating projections asks about the convergence of the sequence $\left(x_{n}\right)_{n \geq 0}$ given by $x_{0}=x, x_{n}=P_{i_{n}} x_{n-1}$. It is an open problem to know whether $\left(x_{n}\right)_{n \geq 0}$ is always convergent in the topology of $H$. The convergence of $\left(x_{n}\right)_{n \geq 0}$ in the weak topology was proved by Amemiya and Andô in [1. If each $j$ between 1 and $N$ occurs infinitely many times in the sequence of random samples, then the weak limit of $\left(x_{n}\right)_{n \geq 0}$ is $P_{M} x$. We refer to [15, 30, 19] for results related to this problem.

1B. The rate of convergence. It is important for applications to know how fast the algorithm given by the method of alternating projections, and its variations, converge. For $N=2$ a quite complete description of the rate of convergence is known, in terms of the notion of the angle between subspaces.

Definition 1.1 (Friedrichs angle). Let $M_{1}$ and $M_{2}$ be two closed subspaces of the Hilbert space $H$ with intersection $M=M_{1} \cap M_{2}$. The Friedrichs angle between the subspaces $M_{1}$ and $M_{2}$ is defined to be the angle in $[0, \pi / 2]$ whose cosine is given by

$$
c\left(M_{1}, M_{2}\right):=\sup \left\{|\langle x, y\rangle|: x \in M_{1} \cap M^{\perp} \cap B_{H}, y \in M_{2} \cap M^{\perp} \cap B_{H}\right\},
$$

where $B_{H}:=\{h \in H:\|h\| \leq 1\}$ is the unit ball of $H$. The minimal angle (or Dixmier angle) between the subspaces $M_{1}$ and $M_{2}$ is defined to be the angle in $[0, \pi / 2]$ whose cosine is given by

$$
c_{0}\left(M_{1}, M_{2}\right):=\sup \left\{|\langle x, y\rangle|: x \in M_{1} \cap B_{H}, y \in M_{2} \cap B_{H}\right\} .
$$

We note that $c\left(M_{1}, M_{2}\right)=c_{0}\left(M_{1} \cap M^{\perp}, M_{2} \cap M^{\perp}\right)$, and that $c_{0}\left(M_{1}, M_{2}\right)=1$ if $M \neq\{0\}$. We also have $c\left(M_{1}, M_{2}\right)=c\left(M_{1}^{\perp}, M_{2}^{\perp}\right)$. We refer to the survey paper 12. for more information about different notions of the angle between subspaces of infinitedimensional Hilbert spaces and their properties, and to [28, Lecture VIII] for various occurrences of the Friedrichs angle in functional-theoretical problems.

It was proved by Aronszajn [2] (upper bound) and by Kayalar and Weinert [17] (equality) that

$$
\left\|\left(P_{M_{2}} P_{M_{1}}\right)^{n}-P_{M}\right\|=c\left(M_{1}, M_{2}\right)^{2 n-1} \quad(n \geq 1) .
$$

This formula shows that the sequence $\left(T^{n}\right)$ of iterates of $T=P_{M_{2}} P_{M_{1}}$ converges uniformly to $T^{\infty}=P_{M}$ if and only if $c\left(M_{1}, M_{2}\right)<1$, i.e., if the Friedrichs angle between $M_{1}$ and $M_{2}$ is positive. When this happens, the iterates of $T=P_{M_{2}} P_{M_{1}}$ converge "quickly" (i.e., at the rate of a geometrical progression) to $T^{\infty}=P_{M}$, in the following sense:

(QUC) (quick uniform convergence) there exist $C>0$ and $\alpha \in] 0,1[$ such that

$$
\left\|T^{n}-T^{\infty}\right\| \leq C \alpha^{n} \quad(n \geq 1) .
$$

It is also known 12 that $c\left(M_{1}, M_{2}\right)<1$ if and only if $M_{1}+M_{2}$ is closed, if and only if $M_{1}^{\perp}+M_{2}^{\perp}$ is closed, if and only if $\left(M_{1} \cap M^{\perp}\right)+\left(M_{2} \cap M^{\perp}\right)$ is closed.

When $M_{1}+M_{2}$ is not closed, we have strong, but not uniform convergence. It was recently proved by Bauschke, Deutsch, and Hundal (see [5] for the history of this result) that, given any sequence of reals decreasing to zero, there exists a point in the Hilbert space with the property that the convergence in the method of alternating projections (von Neumann's theorem) is at least as slow as this sequence of reals. Thus, the iterates of the product of two orthogonal projections converge quickly, or arbitrarily slowly. We call this alternative the (QUC)/(ASC) dichotomy: one has quick uniform convergence or 
arbitrarily slow convergence. We shall consider several meanings of (ASC) in this paper. The results concerning the rate of convergence in Halperin's theorem for $N \geq 3$ are not as complete as the results described above for $N=2$. We refer to [11, 13, 32, [10, Chapter 9 ] and the references therein for several results concerning the rate of convergence in the method of cyclic alternating projections. For instance, [13, Example 3.7] shows that for $N \geq 3$ the error bound for the method of cyclic alternating projections is not a function of the various Friedrichs angles $c\left(M_{i}, M_{j}\right)$ between pairs of subspaces.

1C. What this paper is about. Our main goal in the present paper is to discuss the rate of convergence in Halperin's theorem and to generalize some of the previously known results $(N=2)$ to the case of several subspaces $(N \geq 3)$. We show by operator-theoretical methods that the (QUC)/(ASC) dichotomy always holds as soon as the iterates of $T$ are strongly convergent. Several interpretations of (ASC) are proposed, and general dichotomy theorems are obtained in the Hilbert or Banach space situation, depending on several spectral requirements imposed upon the operator $T$. This implies at once the dichotomy (QUC)/(ASC) in all above-mentioned generalizations of the method of alternating projections. We also give a generalization of the Friedrichs angle to several subspaces, $c\left(M_{1}, \ldots, M_{N}\right)$, and prove that the (QUC) condition is fulfilled in Halperin's theorem if and only if $c\left(M_{1}, \ldots, M_{N}\right)<1$. Estimates for the error $\left\|\left(P_{M_{N}} \ldots P_{M_{2}} P_{M_{1}}\right)^{n}-P_{M}\right\|$ are given in this case, and several statements equivalent to the condition $c\left(M_{1}, \ldots, M_{N}\right)<1$ are obtained. Some of them are expressed in terms of the random products $P_{i_{k}} \ldots P_{i_{1}}$ of projections. More specific descriptions of these results, and information about how the paper is organized, are given below.

1D. Conditions for arbitrarily slow convergence. Several dichotomy theorems of the type quick uniform convergence versus arbitrarily slow convergence are proved in this paper. The quick uniform condition is the condition (QUC) presented above. In $\S 2$ we shall consider the following conditions for (ASC):

(ASC1) (arbitrarily slow convergence, version 1) For every $\varepsilon>0$ and every sequence $\left(a_{n}\right)_{n \geq 1}$ of positive numbers such that $\lim _{n \rightarrow \infty} a_{n}=0$, there exists a vector $x \in X$ such that $\|x\|<\sup _{n} a_{n}+\varepsilon$ and $\left\|T^{n} x-T^{\infty} x\right\| \geq a_{n}$ for all $n$.

(ASC2) (arbitrarily slow convergence, version 2) For every sequence $\left(a_{n}\right)_{n \geq 1}$ of positive numbers such that $\lim _{n \rightarrow \infty} a_{n}=0$, there exists a dense subset of points $x \in X$ such that $\left\|T^{n} x-T^{\infty} x\right\| \geq a_{n}$ for all but a finite number of $n$ 's.

(ASC3) (arbitrarily slow convergence, version 3) For every sequence $\left(a_{n}\right)_{n \geq 1}$ of positive numbers such that $\lim _{n \rightarrow \infty} a_{n}=0$, there exist two vectors $x \in X$ and $y \in X^{*}$ (the dual of $X$ ) such that $\operatorname{Re}\left\langle T^{n} x-T^{\infty} x, y\right\rangle \geq a_{n}$ for all $n \geq 1$. Furthermore, if there is a Banach space $Y$ such that $X$ is a (isometrical) subspace of $Y^{*}$, then the vector $y$ can be chosen in $Y$.

(ASCH) (arbitrarily slow convergence, Hilbertian version) For every $\varepsilon>0$ and every sequence $\left(a_{n}\right)_{n \geq 1}$ of positive numbers such that $\lim _{n \rightarrow \infty} a_{n}=0$, there exists a vector $x \in H$ such that $\|x\|<\sup _{n} a_{n}+\varepsilon$ and $\operatorname{Re}\left\langle T^{n} x-T^{\infty} x, x\right\rangle \geq a_{n}$ for all $n \geq 1$. Here $H$ is assumed to be a complex Hilbert space.

The dichotomy results of $\S 2$ are based upon general results about the existence of large (weak) orbits of operators (see [26, 24, 25]).

Here we recall the main result of 25 concerning large weak orbits in the Banach space setting.

Theorem 1.2 (see [25]). Let $X$ be a Banach space that does not contain $c_{0}$, and $T$ a bounded operator on $X$ such that 1 belongs to the spectrum of $T$ and $\left\|T^{n} x\right\|$ tends to zero for every $x \in X$ as $n$ tends to infinity. Then for any sequence $\left(a_{n}\right)_{n \geq 0}$ such that $a_{n}$ tends 
to zero as $n$ tends to infinity, there exists a vector $x \in X$ and a functional $x^{*} \in X^{*}$ such that $\operatorname{Re}\left\langle T^{n} x, x^{*}\right\rangle \geq a_{n}$ for every $n \geq 0$.

We prove in $\S 2$ that if the iterates of $T \in \mathcal{B}(X)$ are strongly convergent, then we have (QUC) or (ASC1). Also, if the iterates are strongly convergent, then the dichotomy (QUC)/(ASC2) holds. Condition (QUC) is fulfilled if and only if $\operatorname{Ran}(\lambda I-T)$, the range of $\lambda I-T$, is closed for each $\lambda$ in the unit circle $\partial \mathbf{D}$. In the case where $T \in \mathcal{B}(X)$ is a power bounded, mean ergodic operator with spectrum $\sigma(T)$ included in $\mathbb{D} \cup\{1\}$, we use the Katznelson-Tzafriri theorem [20] to prove that the iterates of $T$ are strongly convergent. Therefore, the previous dichotomies (QUC)/(ASC1) and (QUC)/(ASC2) apply. Moreover, the dichotomy (QUC)/(ASC3) holds whenever the Banach space $X$ contains no isomorphic copy of $c_{0}$. If $X=H$ is a Hilbert space, then also the dichotomy (QUC)/(ASCH) holds. We prove here that the (QUC) condition is fulfilled if and only if $\operatorname{Ran}(I-T)$ is closed. Applications to products of projections of norm one are given. In particular, the dichotomy (QUC)/(ASC) holds, with several versions of (ASC), for the cases covered by the theorems of von Neumann, Halperin, Bruck-Reich, and those of [3].

1E. A generalization of the Friedrichs angle. In order to quantify the rate of convergence in the method of alternating projections, an extension of the cosine of Friedrichs angle to several subspaces $\left(M_{1}, \ldots, M_{N}\right)$ will be given in $\S 3$. It is a parameter $c\left(M_{1}, \ldots, M_{N}\right)$ that lies between 0 and 1 and is defined as follows:

$$
\begin{aligned}
& c\left(M_{1}, \ldots, M_{N}\right) \\
& \quad=\sup \left\{\frac{2}{N-1} \frac{\sum_{j<k} \operatorname{Re}\left\langle m_{j}, m_{k}\right\rangle}{\left\|m_{1}\right\|^{2}+\cdots+\left\|m_{N}\right\|^{2}}: m_{j} \in M_{j} \cap M^{\perp},\left\|m_{1}\right\|^{2}+\cdots+\left\|m_{N}\right\|^{2} \neq 0\right\} \\
& \quad=\sup \left\{\frac{1}{N-1} \frac{\sum_{j \neq k}\left\langle m_{j}, m_{k}\right\rangle}{\sum_{j=1}^{N}\left\langle m_{j}, m_{j}\right\rangle}: m_{j} \in M_{j} \cap M^{\perp},\left\|m_{1}\right\|^{2}+\cdots+\left\|m_{N}\right\|^{2} \neq 0\right\} .
\end{aligned}
$$

The fact that this definition coincides with the classical one for two subspaces will be proved in Lemma 3.1. For $N$ pairwise orthogonal subspaces (the "angle" is $\pi / 2$ in this case) we have $c\left(M_{1}, \ldots, M_{N}\right)=0$, while the other extremal case $c\left(M_{1}, \ldots, M_{N}\right)=1$ corresponds to arbitrarily slow convergence in the method of cyclic alternating projections for $N$ subspaces (the "angle" is zero). Notice that the definition of the Friedrichs parameter excludes the degenerate case where all subspaces $M_{j}$ coincide. Other related quantities are considered: the configuration constant $\kappa\left(M_{1}, \ldots, M_{N}\right)$, the inclination $\ell\left(M_{1}, \ldots, M_{N}\right)$, and the Friedrichs angle between the Cartesian product $\mathbf{C}=M_{1} \times \cdots \times$ $M_{N} \subset H^{N}$ and the "diagonal subspace" $\mathbf{D}=\operatorname{diag}(H)=\{(y, \ldots, y): y \in H\} \subset H^{N}$.

In $\S 4$ we characterize in several ways when the dichotomy (QUC)/(ASC) arises. The characterizations are in terms of geometric properties of $\left(M_{1}, \ldots, M_{N}\right)$, of spectral properties of $T$, or of random products $P_{i_{k}} \ldots P_{i_{1}}$. Also, we give an estimate for the geometric convergence of $\left\|T^{n}-P_{M}\right\|$ to zero when $c\left(M_{1}, \ldots, M_{N}\right)<1$.

\section{§2. General Dichotomy theorems and applichtions}

\section{A. Dichotomy theorems.}

Theorem 2.1 ((QUC)/(ASC1) and (QUC)/(ASC2)). Let $X$ be a Banach space and let $T \in \mathcal{B}(X)$ be such that the sequence of iterates $\left(T^{n}\right)$ is strongly convergent to $T^{\infty} \in \mathcal{B}(X)$. Then the following dichotomy holds: either (QUC), or (ASC1) is true. The quick uniform convergence (condition (QUC)) holds if and only if

$$
\text { for every } \lambda \in \partial \mathbf{D}, \quad \operatorname{Ran}(\lambda-T) \text { is closed. }
$$

In these statements, condition (ASC1) can be replaced by (ASC2). 
Proof. Suppose that the sequence of iterates $\left(T^{n}\right)_{n \geq 0}$ is strongly convergent to $T^{\infty} \in$ $\mathcal{B}(X)$. Then $T$ is mean ergodic, i.e., the Cesàro means $\left(I+T+\cdots+T^{n-1}\right) / n$ are strongly convergent. Therefore (see [21, p. 73]), the space $X$ can be decomposed as the direct sum of the kernel of $T-I$ and the closure of the range of the same operator, $X=\operatorname{Ker}(T-I) \oplus \overline{\operatorname{Ran}(T-I)}$. Moreover, $T^{\infty}$ is the projection onto $\operatorname{Ker}(T-I)$ along $\overline{\operatorname{Ran}(T-I)}$. Notice also that $T^{\infty}$ acts on the space $\operatorname{Ker}(T-I)$ as the identity. With respect to the decomposition $X=\operatorname{Ker}(T-I) \oplus \overline{\operatorname{Ran}(T-I)}$ we can write

$$
T=\left(\begin{array}{cc}
T^{\infty} & 0 \\
0 & A
\end{array}\right)
$$

for some $A \in \mathcal{B}(\overline{\operatorname{Ran}(T-I)})$. It is not difficult to prove that for every $\lambda \in \mathbb{C}$, the range $\operatorname{Ran}(T-\lambda I)$ is closed if and only if $\operatorname{Ran}(A-\lambda I)$ is. The strong convergence of $T^{n}$ and the Banach-Steinhaus theorem imply that $T$ is power bounded, i.e., $\sup _{n>1}\left\|T^{n}\right\|<\infty$. Thus $\sigma(T)$, the spectrum of $T$, is included in the closed unit disk. Since $\sigma(\bar{T})=\{1\} \cup \sigma(A)$, the same inclusion is true for $\sigma(A)$. In particular, the spectral radius of $A$ satisfies $r(A) \leq 1$.

We distinguish two cases.

Case 1 . We have $r(A)<1$. Observe that

$$
T^{n}-T^{\infty}=\left(\begin{array}{cc}
0 & 0 \\
0 & A^{n}
\end{array}\right) .
$$

Since $r(A)<1$, there exists $C>0$ and $\alpha \in] 0,1[$ such that

$$
\left\|A^{n}\right\| \leq C \alpha^{n} \quad(n \geq 1) .
$$

This estimate and (2.2) give the quick uniform convergence condition (QUC).

Case 2. We have $r(A)=1$. Recall that $\left\|A^{n} y\right\| \rightarrow 0$ as $n \rightarrow \infty$, for each $y \in \overline{\operatorname{Ran}(T-I)}$. Conditions (ASC1) and (ASC2) follow now from [26, Theorem 14, p. 333].

Suppose that Case (1) is fulfilled, i.e., $r(A)<1$. Then $A-\lambda$ is invertible for every $\lambda \in \partial \mathbf{D}$. In particular, $\operatorname{Ran}(A-\lambda)=\overline{\operatorname{Ran}(T-I)}$ is closed for each $\lambda \in \partial \mathbf{D}$. Thus, $\operatorname{Ran}(T-\lambda)$ is also closed for each $\lambda \in \partial \mathbf{D}$.

Suppose now that all subspaces $\operatorname{Ran}(T-\lambda), \lambda \in \partial \mathbf{D}$, and so all $\operatorname{Ran}(A-\lambda), \lambda \in \partial \mathbf{D}$, are closed. Then $r(A)<1$. Indeed, suppose that $r(A)=1$ and let $\lambda \in \partial \mathbf{D} \cap \sigma(A)$ be a point in the unimodular spectrum of $A$. Then the condition $\left\|A^{n} y\right\| \rightarrow 0$ as $n \rightarrow \infty$ for each $y$ shows that $\lambda$ cannot be an eigenvalue: if $A y=\lambda y$, then $y=0$. Indeed, we have $\|y\|=\left\|\lambda^{-n} A^{n} y\right\|=\left\|A^{n} y\right\| \rightarrow 0$ as $n \rightarrow \infty$. Thus, $\lambda \in \sigma(A) \backslash \sigma_{p}(A)$ and $\operatorname{Ran}(A-\lambda)$ is closed. Therefore, $A-\lambda$ is an upper semi-Fredholm operator. Since $A-\lambda I$ is the limit of the invertible operators $A-\frac{n+1}{n} \lambda I$, the index $\operatorname{ind}(A-\lambda I)$ of $A-\lambda I$ is 0 . Hence, $A-\lambda I$ is invertible, which contradicts the assumption that $\lambda \in \sigma(A)$. Thus, $r(A)<1$.

Remark 2.2. The following is a different argument for the last part of the proof, without the use of Fredholm theory. Since $\lambda \in \sigma(A) \backslash \sigma_{p}(A)$ and $\operatorname{Ran}(A-\lambda)$ is closed, the operator $A-\lambda$ is lower bounded, so that $\lambda$ is not in the approximate point spectrum of $A$. Since every point in the boundary of the spectrum is in the approximate point spectrum, we obtain the desired contradiction. We refer the reader to [26] as a basic reference for the spectral theory of linear operators we are using in the present paper.

Theorem 2.3 ((QUC)/(ASC3) and (QUC)/(ASCH)). Let $X$ be a Banach space, and let $T \in \mathcal{B}(X)$ be a power bounded, mean ergodic operator with spectrum $\sigma(T)$ included in $\mathbb{D} \cup\{1\}$. Then the sequence of iterates $T^{n}$ is strongly convergent to a certain operator $T^{\infty} \in \mathcal{B}(X)$, and the dichotomies of Theorem 2.1 apply. Moreover, the dichotomy (QUC)/(ASC3) holds whenever $X$ contains no isomorphic copy of $c_{0}$. If $X=H$ is a Hilbert space, then also the dichotomy (QUC)/(ASCH) holds. 
In all these statements, the quick uniform convergence condition (QUC) holds if and only if

$$
\operatorname{Ran}(I-T) \text { is closed. }
$$

Proof. Again, by using mean ergodicity and [21, p. 73], the space $X$ can be decomposed as the direct sum $X=\operatorname{Ker}(T-I) \oplus \overline{\operatorname{Ran}(T-I)}$. By the Katznelson-Tzafriri theorem [20], the power boundedness condition and the spectral condition $\sigma(T) \subset \mathbb{D} \cup\{1\}$ imply $\lim _{n \rightarrow \infty}\left\|T^{n+1}-T^{n}\right\|=0$. This shows that the sequence of iterates $\left(T^{n}\right)$ of $T$ converges strongly to 0 on the range of $T-I$. The same is true for the closure $\overline{\operatorname{Ran}(T-I)}$. Since $T$ acts like identity on $\operatorname{Ker}(T-I)$, we see that $\left(T^{n}\right)_{n \geq 0}$ converges strongly to $T^{\infty}$, the projection onto $\operatorname{Ker}(T-I)$ along $\overline{\operatorname{Ran}(T-I)}$. Thus, we can apply Theorem 2.1 to obtain the dichotomies (QUC)/(ASC1) and (QUC)/(ASC2).

Now we show that (QUC)/(ASC3) also holds if $X$ contains no isomorphic copy of $c_{0}$. With the notation of the proof of Theorem 2.1, if the condition (QUC) is not satisfied, then $r(A)=1$ (Case (2) in the proof of Theorem 2.1). Since $\sigma(T) \subset \mathbb{D} \cup\{1\}$, we have the same inclusion for the spectrum of $A$. Therefore, $1 \in \sigma(A)$. Observe also that $\left\|A^{n} y\right\| \rightarrow 0$ as $n \rightarrow \infty$, because $\left(T^{n}\right)$ converges strongly to $T^{\infty}$. Now we can apply Theorem 1.2 provided that $X$ contains no isomorphic copy of $c_{0}$. To obtain the dichotomy (QUC)/(ASCH) if $X=H$ is a Hilbert space, we use [24, Theorem 2] (see also [4, Theorem 1] for the case of weak convergence).

2B. Applications to the method of alternating projections. First we introduce some notation and, for the reader's convenience, recall some Banach space terminology. Let $N \geq 2$. Let $X$ be a Banach space, and let $P_{1}, \ldots, P_{N}$ be $N$ fixed projections $\left(P_{j}^{2}=P_{j}\right)$ acting on $X$. We denote by $\mathcal{S}=\mathcal{S}\left(P_{1}, \ldots, P_{N}\right)$ the convex multiplicative semigroup generated by $P_{1}, \ldots, P_{N}$. Recall that this is the convex hull of the set of all products with factors taken among $P_{1}, \ldots, P_{N}$, and that the convex hull of every multiplicative semigroup of operators is a semigroup.

The space $X$ is said to be uniformly convex if for every $\varepsilon \in(0,1)$ there exists $\delta \in$ $(0,1)$ such that for any two vectors $x$ and $y$ with $\|x\| \leq 1$ and $\|y\| \leq 1$, the inequality $\|x+y\| / 2>1-\delta$ implies $\|x-y\|<\varepsilon$. An (equivalent) definition of a uniformly smooth Banach space is the following: $X$ is uniformly smooth if its dual $X^{*}$ is uniformly convex. We refer to 23] for more information.

We call $P \in \mathcal{B}(X)$ a norm one projection (nonzero orthoprojection) if $P^{2}=P$ and $\|P\|=1$. As usual, a selfadjoint projection in a Hilbert space is called an orthogonal projection. Recall that an operator $T$ on a Banach space $X$ is called Hermitian if its numerical range is real. This is equivalent to the fact that $\|\exp (i t T)\|=1$ for every real $t$. The Hermitian operators on Hilbert spaces coincide with the selfadjoint ones; see for instance [8] and the references therein.

Theorem 2.4. Let $N \geq 2$. Let $X$ be a complex Banach space, and let $P_{1}, \ldots, P_{N}$ be $N$ projections on $X$. Let $T$ be an operator in $\mathcal{S}\left(P_{1}, \ldots, P_{N}\right)$. If one of the following conditions below is fulfilled, then the sequence of iterates of $T$ converges strongly, and every dichotomy (QUC)/(ASC1), (QUC)/(ASC2), (QUC)/(ASC3), and (QUC)/(ASCH) (if $X=H$ is a Hilbert space) applies:

(i) The space $X$ is uniformly convex and each $P_{j}, 1 \leq j \leq N$, is a norm one projection.

(ii) The space $X$ is uniformly smooth, and each $P_{j}, 1 \leq j \leq N$, is a norm one projection. 
(iii) The space $X$ is reflexive and for each $j$ there exists $r_{j}$ with $0<r_{j}<1$ such that $\left\|P_{j}-r_{j} I\right\| \leq 1-r_{j}$. In particular, this is true if each $P_{j}$ is Hermitian, $1 \leq j \leq N$.

Proof. In [3] it was proved that in all three situations the spectrum of $T \in \mathcal{S}\left(P_{1}, \ldots, P_{N}\right)$ is included in $\mathbb{D} \cup\{1\}$ and that the iterates of $T$ are strongly convergent. We apply the above dichotomy theorems. Notice that uniformly convex and uniformly smooth Banach spaces are reflexive, and that reflexive Banach spaces contain no copies of $c_{0}$.

\section{§3. A generalization of the Friedrichs angle for $N$ subspaces}

As was mentioned in the Introduction, the rate of convergence in the method of alternating projections for two closed subspaces $M_{1}$ and $M_{2}$ is controlled by the Friedrichs angle $c\left(M_{1}, M_{2}\right)$. In this section we introduce and study a generalization of the Friedrichs angle for $N$ subspaces.

3A. Definition. In order to introduce our generalization of the cosine of the Friedrichs angle to several closed subspaces, we start by giving an equivalent definition of the Friedrichs angle $c\left(M_{1}, M_{2}\right)$.

Lemma 3.1. (a) Let $M_{1}$ and $M_{2}$ be two closed subspaces of $H$. Then

$$
c_{0}\left(M_{1}, M_{2}\right)=\sup \left\{\frac{2 \operatorname{Re}\left\langle m_{1}, m_{2}\right\rangle}{\left\|m_{1}\right\|^{2}+\left\|m_{2}\right\|^{2}}: m_{1} \in M_{1}, m_{2} \in M_{2},\left(m_{1}, m_{2}\right) \neq(0,0)\right\} .
$$

(b) Let $M_{1}$ and $M_{2}$ be two closed subspaces in $H$. Then

$$
\begin{aligned}
c\left(M_{1}, M_{2}\right) & =\sup \left\{\frac{2 \operatorname{Re}\left\langle m_{1}, m_{2}\right\rangle}{\left\|m_{1}\right\|^{2}+\left\|m_{2}\right\|^{2}}: m_{j} \in M_{j} \cap M^{\perp},\left(m_{1}, m_{2}\right) \neq(0,0)\right\} \\
& =\sup \left\{\frac{\left\langle m_{1}, m_{2}\right\rangle+\left\langle m_{2}, m_{1}\right\rangle}{\left\langle m_{1}, m_{1}\right\rangle+\left\langle m_{2}, m_{2}\right\rangle}: m_{j} \in M_{j} \cap M^{\perp},\left(m_{1}, m_{2}\right) \neq(0,0)\right\} .
\end{aligned}
$$

Proof. We give the proof only for the first identity of part (b). Denote by $s$ the first supremum in the statement of part (b). For every admissible pair $\left(m_{1}, m_{2}\right)$ with $\left(m_{1}, m_{2}\right) \neq(0,0)$, we have

$$
\frac{2}{\left\|m_{1}\right\|^{2}+\left\|m_{2}\right\|^{2}} \operatorname{Re}\left\langle m_{1}, m_{2}\right\rangle \leq \frac{1}{\left\|m_{1}\right\| \cdot\left\|m_{2}\right\|} \operatorname{Re}\left\langle m_{1}, m_{2}\right\rangle \leq \frac{\left|\left\langle m_{1}, m_{2}\right\rangle\right|}{\left\|m_{1}\right\| \cdot\left\|m_{2}\right\|} \leq c\left(M_{1}, M_{2}\right) .
$$

Therefore, $s \leq c\left(M_{1}, M_{2}\right)$.

For the reverse inequality, let $\varepsilon>0$. Then there exist two elements $x_{1} \in M_{1} \cap M^{\perp}$ and $x_{2} \in M_{2} \cap M^{\perp}$ with $\left\|x_{1}\right\|=1$ and $\left\|x_{2}\right\|=1$ such that $c\left(M_{1}, M_{2}\right)<\left|\left\langle x_{1}, x_{2}\right\rangle\right|+\varepsilon$. Let $\theta \in \mathbb{R}$ be such that $\left\langle x_{1}, x_{2}\right\rangle=\left|\left\langle x_{1}, x_{2}\right\rangle\right| e^{i \theta}$, and set $m_{1}=e^{-i \theta} x_{1}$ and $m_{2}=x_{2}$. Then $m_{1} \in M_{1} \cap M^{\perp}, m_{2} \in M_{2} \cap M^{\perp}$ and $\left\|m_{1}\right\|=1,\left\|m_{2}\right\|=1$. We obtain

$$
s \geq \frac{2 \operatorname{Re}\left\langle m_{1}, m_{2}\right\rangle}{\left\|m_{1}\right\|^{2}+\left\|m_{2}\right\|^{2}}=\operatorname{Re}\left\langle e^{-i \theta} x_{1}, x_{2}\right\rangle=\left|\left\langle x_{1}, x_{2}\right\rangle\right|>c\left(M_{1}, M_{2}\right)-\varepsilon .
$$

Since $\varepsilon$ is arbitrary, we obtain $s=c\left(M_{1}, M_{2}\right)$.

Definition 3.2. Let $N \geq 2$. Let $M_{1}, \ldots, M_{N}$ be $N$ closed subspaces of $H$ with intersection $M=M_{1} \cap \cdots \cap M_{N}$. The Dixmier number associated with $\left(M_{1}, \ldots, M_{N}\right)$ is defined as

$$
\begin{aligned}
& c_{0}\left(M_{1}, \ldots, M_{N}\right) \\
& \quad=\sup \left\{\frac{2}{N-1} \frac{\sum_{j<k} \operatorname{Re}\left\langle m_{j}, m_{k}\right\rangle}{\left\|m_{1}\right\|^{2}+\cdots+\left\|m_{N}\right\|^{2}}: m_{j} \in M_{j},\left\|m_{1}\right\|^{2}+\cdots+\left\|m_{N}\right\|^{2} \neq 0\right\} .
\end{aligned}
$$


The Friedrichs number $c\left(M_{1}, \ldots, M_{N}\right)$ associated with $\left(M_{1}, \ldots, M_{N}\right)$ is defined as

$$
\begin{aligned}
& c\left(M_{1}, \ldots, M_{N}\right) \\
& \quad=\sup \left\{\frac{2}{N-1} \frac{\sum_{j<k} \operatorname{Re}\left\langle m_{j}, m_{k}\right\rangle}{\left\|m_{1}\right\|^{2}+\cdots+\left\|m_{N}\right\|^{2}}: m_{j} \in M_{j} \cap M^{\perp},\left\|m_{1}\right\|^{2}+\cdots+\left\|m_{N}\right\|^{2} \neq 0\right\} \\
& \quad=\sup \left\{\frac{1}{N-1} \frac{\sum_{j \neq k}\left\langle m_{j}, m_{k}\right\rangle}{\sum_{j=1}^{N}\left\langle m_{j}, m_{j}\right\rangle}: m_{j} \in M_{j} \cap M^{\perp},\left\|m_{1}\right\|^{2}+\cdots+\left\|m_{N}\right\|^{2} \neq 0\right\} .
\end{aligned}
$$

3B. Other parameters, and properties of the Friedrichs number. We found it convenient to introduce the following parameters, called the (reduced or not) configuration constants, although they can be expressed in terms of the Dixmier and Friedrichs numbers (see Proposition [3.6. (f)).

Definition 3.3. Let $N \geq 2$. Let $M_{1}, \ldots, M_{N}$ be $N$ closed subspaces of $H$ with intersection $M=M_{1} \cap \cdots \cap M_{N}$. The number

$$
\kappa_{0}\left(M_{1}, \ldots, M_{N}\right)=\sup \left\{\frac{1}{N} \frac{\left\|\sum_{j=1}^{N} m_{j}\right\|^{2}}{\sum_{j=1}^{N}\left\|m_{j}\right\|^{2}}: m_{j} \in M_{j},\left\|m_{1}\right\|^{2}+\cdots+\left\|m_{N}\right\|^{2} \neq 0\right\}
$$

is called the nonreduced configuration constant of $\left(M_{1}, \ldots, M_{N}\right)$. The number

$$
\kappa\left(M_{1}, \ldots, M_{N}\right)=\sup \left\{\frac{1}{N} \frac{\left\|\sum_{j=1}^{N} m_{j}\right\|^{2}}{\sum_{j=1}^{N}\left\|m_{j}\right\|^{2}}: m_{j} \in M_{j} \cap M^{\perp},\left\|m_{1}\right\|^{2}+\cdots+\left\|m_{N}\right\|^{2} \neq 0\right\}
$$

is called the configuration constant of $\left(M_{1}, \ldots, M_{N}\right)$.

The configuration constant is related to the maximal possible norms of Gramian matrices. Recall that the Gramian matrix of an $N$-tuple of vectors $\left(v_{1}, \ldots, v_{N}\right)$ is the $(N \times N)$-matrix

$$
G\left(v_{1}, \ldots, v_{N}\right)=\left[\left\langle v_{i}, v_{j}\right\rangle\right]_{1 \leq i, j \leq N} .
$$

Proposition 3.4. Let $N \geq 2$. Let $M_{1}, \ldots, M_{N}$ be $N$ closed subspaces of $H$ with intersection $M=M_{1} \cap \cdots \cap M_{N}$. Then

$$
\kappa\left(M_{1}, \ldots, M_{N}\right)=\sup \left\{\frac{1}{N}\left\|G\left(v_{1}, \ldots, v_{N}\right)\right\|: v_{j} \in M_{j} \cap M^{\perp},\left\|v_{j}\right\|=1, j=1, \ldots, N\right\} .
$$

Proof. Let $m_{j} \in M_{j} \cap M^{\perp}, j=1, \ldots, N$, with $\left\|m_{1}\right\|^{2}+\cdots+\left\|m_{N}\right\|^{2} \neq 0$. Set $v_{j}=\frac{m_{j}}{\left\|m_{j}\right\|}$ if $\left\|m_{j}\right\| \neq 0$, or $v_{j}=0$ if $m_{j}=0$. Denote $\mathbf{x}=\left(\left\|m_{1}\right\|, \ldots,\left\|m_{N}\right\|\right) \in \mathbb{C}^{N} \backslash\{0\}$. We have $\langle\mathbf{x}, \mathbf{x}\rangle=\mathbf{x}^{t} \mathbf{x} \neq 0$ and

$$
\frac{1}{N} \frac{\left\|\sum_{j=1}^{N} m_{j}\right\|^{2}}{\sum_{j=1}^{N}\left\|m_{j}\right\|^{2}}=\frac{1}{N} \frac{\left\langle\sum_{i}^{N}\left\|m_{i}\right\| v_{i}, \sum_{j}^{N}\left\|m_{j}\right\| v_{j}\right\rangle}{\sum_{j=1}^{N}\left\|m_{j}\right\|^{2}}=\frac{1}{N} \frac{\mathbf{x}^{t} G\left(v_{1}, \ldots, v_{N}\right) \mathbf{x}}{\mathbf{x}^{t} \mathbf{x}} .
$$

The conclusion follows by taking the supremum and noting that the Gramian matrix $G\left(v_{1}, \ldots, v_{N}\right)$ is a Hermitian matrix.

Consider the product Hilbert space $H^{N}$, which is the Hilbertian direct sum of $N$ copies of $H$, with the scalar product

$$
\langle\mathbf{x}, \mathbf{y}\rangle=\left\langle\left(x_{1}, \ldots, x_{N}\right),\left(y_{1}, \ldots, y_{N}\right)\right\rangle:=\sum_{j=1}^{N}\left\langle x_{j}, y_{j}\right\rangle .
$$

We denote by $\mathbf{C}$ the Cartesian product $\mathbf{C}=M_{1} \times \cdots \times M_{N} \subset H^{N}$, and by $\mathbf{D}$ the diagonal subset $\mathbf{D}=\operatorname{diag}(H)=\{(y, \ldots, y): y \in H\} \subset H^{N}$. Recall that $M=M_{1} \cap \cdots \cap M_{N}$. 
Lemma 3.5. The projections onto $\mathbf{C}, \mathbf{D}$, and $\mathbf{C} \cap \mathbf{D}$ are given by

$$
\begin{aligned}
& P_{\mathbf{C}}\left(x_{1}, \ldots, x_{N}\right)=\left(P_{1} x_{1}, \ldots, P_{N} x_{N}\right), \\
& P_{\mathbf{D}}\left(x_{1}, \ldots, x_{N}\right)=\left(\left(x_{1}+\cdots+x_{N}\right) / N, \ldots,\left(x_{1}+\cdots+x_{N}\right) / N\right),
\end{aligned}
$$

and, respectively, by

$$
P_{\mathbf{C} \cap \mathbf{D}}\left(x_{1}, \ldots, x_{N}\right)=\left(\left(P_{M} x_{1}+\cdots+P_{M} x_{N}\right) / N, \ldots,\left(P_{M} x_{1}+\cdots+P_{M} x_{N}\right) / N\right),
$$

where $\left(x_{1}, \ldots, x_{N}\right) \in H^{N}$.

Proof. The formulas for $P_{\mathbf{C}}$ and $P_{\mathbf{D}}$ were proved in 29 . For the third one, first we note that

$$
\mathbf{C} \cap \mathbf{D}=\operatorname{diag}(M)=\{(m, \ldots, m): m \in M\} .
$$

Therefore,

We obtain

$$
\left\|\mathbf{x}-P_{\mathbf{C} \cap \mathbf{D}} \mathbf{x}\right\|^{2}=\operatorname{dist}(\mathbf{x}, \operatorname{diag}(M))^{2}=\inf \left\{\sum_{j=1}^{N}\left\|x_{j}-m\right\|^{2}: m \in M\right\} .
$$

$$
\left\|\mathbf{x}-P_{\mathbf{C} \cap \mathbf{D}} \mathbf{x}\right\|^{2}=\sum_{j=1}^{N}\left\|x_{j}-P_{M} x_{j}\right\|^{2}+\inf \left\{\sum_{j=1}^{N}\left\|P_{M} x_{j}-m\right\|^{2}: m \in M\right\} .
$$

The infimum is realized when the gradient is zero, i.e., $\sum_{j=1}^{N}\left(m-P_{M} x_{j}\right)=0$, that is, when $m=N^{-1} \sum_{j=1}^{N} P_{M} x_{j}$.

Proposition 3.6. Let $N \geq 2$. Let $M_{1}, \ldots, M_{N}$ be $N$ closed subspaces of $H$ with intersection $M=M_{1} \cap \cdots \cap M_{N}$. Then:

(a) $c_{0}\left(M_{1}, \ldots, M_{N}\right)=1$ if $M \neq\{0\}$, while $c_{0}\left(M_{1}, \ldots, M_{N}\right)=0$ if and only if the subspaces $\left(M_{1}, \ldots, M_{N}\right)$ are pairwise orthogonal;

(b) $c\left(M_{1}, \ldots, M_{N}\right)=c\left(M_{1} \cap M^{\perp}, \ldots, M_{N} \cap M^{\perp}\right)=c_{0}\left(M_{1} \cap M^{\perp}, \ldots, M_{N} \cap M^{\perp}\right)$, and thus, $c\left(M_{1}, \ldots, M_{N}\right)=c_{0}\left(M_{1}, \ldots, M_{N}\right)$ if $M=\{0\}$;

(c) $0 \leq c_{0}\left(M_{1}, \ldots, M_{N}\right) \leq 1$ and $0 \leq c\left(M_{1}, \ldots, M_{N}\right) \leq 1$;

(d) $\frac{1}{N} \leq \kappa_{0}\left(M_{1}, \ldots, M_{N}\right) \leq 1$ and $\frac{1}{N} \leq \kappa\left(M_{1}, \ldots, M_{N}\right) \leq 1$;

(e) $\kappa_{0}\left(M_{1}, \ldots, M_{N}\right)=c_{0}(\mathbf{C}, \mathbf{D})^{2}$ and $\kappa\left(M_{1}, \ldots, M_{N}\right)=c(\mathbf{C}, \mathbf{D})^{2}$;

(f) $c\left(M_{1}, \ldots, M_{N}\right)=\frac{N}{N-1} \kappa\left(M_{1}, \ldots, M_{N}\right)-\frac{1}{N-1}=\frac{N}{N-1} c(\mathbf{C}, \mathbf{D})^{2}-\frac{1}{N-1}$ and similar statements hold true for $c_{0}\left(M_{1}, \ldots, M_{N}\right)$.

Proof. We start by giving the proof of part (e). We have

$$
\begin{aligned}
c(\mathbf{C}, \mathbf{D})^{2}= & \sup \left\{\frac{\left|\left\langle\left(m_{1}, \ldots, m_{N}\right),(y, \ldots, y)\right\rangle_{H^{N}}\right|^{2}}{N\left(\left\|m_{1}\right\|^{2}+\cdots+\left\|m_{N}\right\|^{2}\right)\|y\|^{2}}:\right. \\
& \left.y \in H, y \neq 0, m_{j} \in M_{j} \cap M^{\perp},\left\|m_{1}\right\|^{2}+\cdots+\left\|m_{N}\right\|^{2} \neq 0\right\} \\
= & \sup \left\{\frac{\left\langle\sum_{j=1}^{N} m_{j}, y\right\rangle^{2}}{N\left(\left\|m_{1}\right\|^{2}+\cdots+\left\|m_{N}\right\|^{2}\right)\|y\|^{2}}:\right. \\
= & \sup \left\{\frac{\left\|\sum_{j=1}^{N} m_{j}\right\|^{2}}{N\left(\left\|m_{1}\right\|^{2}+\cdots+\left\|m_{N}\right\|^{2}\right)}:\right. \\
\left.y \in H, y \neq 0, m_{j} \in M_{j} \cap M^{\perp},\left\|m_{1}\right\|^{2}+\cdots+\left\|m_{N}\right\|^{2} \neq 0\right\} & \\
= & \kappa\left(M_{1}, \ldots, M_{N}\right) .
\end{aligned}
$$


The proof of the relation $\kappa_{0}\left(M_{1}, \ldots, M_{N}\right)=c_{0}(\mathbf{C}, \mathbf{D})^{2}$ is similar.

Now we prove that $c\left(M_{1}, \ldots, M_{N}\right)=\frac{N}{N-1} \kappa\left(M_{1}, \ldots, M_{N}\right)-\frac{1}{N-1}$. Indeed, we have

$$
\begin{aligned}
c & \left.M_{1}, \ldots, M_{N}\right) \\
& =\sup \left\{\frac{2}{N-1} \frac{\sum_{j<k} \operatorname{Re}\left\langle m_{j}, m_{k}\right\rangle}{\left\|m_{1}\right\|^{2}+\cdots+\left\|m_{N}\right\|^{2}}: m_{j} \in M_{j} \cap M^{\perp},\left\|m_{1}\right\|^{2}+\cdots+\left\|m_{N}\right\|^{2} \neq 0\right\} \\
& =\sup \left\{\frac{1}{N-1} \frac{\left\|\sum_{j} m_{j}\right\|^{2}-\sum_{j}\left\|m_{j}\right\|^{2}}{\left\|m_{1}\right\|^{2}+\cdots+\left\|m_{N}\right\|^{2}}: m_{j} \in M_{j} \cap M^{\perp},\left\|m_{1}\right\|^{2}+\cdots+\left\|m_{N}\right\|^{2} \neq 0\right\} \\
& =\frac{N}{N-1} \kappa\left(M_{1}, \ldots, M_{N}\right)-\frac{1}{N-1} .
\end{aligned}
$$

The proof of the identity for $c_{0}\left(M_{1}, \ldots, M_{N}\right)$ is similar.

The upper bound $\kappa_{0}\left(M_{1}, \ldots, M_{N}\right) \leq 1$ in (d) follows from the Cauchy-Schwarz inequality:

$$
\left\|m_{1}+\cdots+m_{N}\right\|^{2} \leq\left(\left\|m_{1}\right\|+\cdots+\left\|m_{N}\right\|\right)^{2} \leq N\left(\left\|m_{1}\right\|^{2}+\cdots+\left\|m_{N}\right\|^{2}\right) .
$$

For the lower bound $\kappa_{0}\left(M_{1}, \ldots, M_{N}\right) \geq 1 / N$, notice that, for $m_{1} \in M_{1} \backslash\{0\}$, we have

$$
\kappa_{0}\left(M_{1}, \ldots, M_{N}\right) \geq \frac{1}{N} \frac{\left\|m_{1}\right\|^{2}}{\left\|m_{1}\right\|^{2}}=\frac{1}{N} .
$$

The inequalities for $\kappa\left(M_{1}, \ldots, M_{N}\right)$ follow from the relation

$$
\kappa\left(M_{1}, \ldots, M_{N}\right)=\kappa_{0}\left(M_{1} \cap M^{\perp}, \ldots, M_{N} \cap M^{\perp}\right) .
$$

Now (c) is a consequence of (f) and (d), while (b) and (a) are easy to prove. For the first identity in (b), observe that $\bigcap_{j=1}^{N}\left(M_{j} \cap M^{\perp}\right)=\{0\}$.

Proposition 3.7. Let $N \geq 2$. Let $M_{1}, \ldots, M_{N}$ be $N$ closed subspaces of $H$ with intersection $M=M_{1} \cap \cdots \cap M_{N}$. Then

$$
\kappa\left(M_{1}, \ldots, M_{N}\right)=\left\|\frac{P_{1}+\cdots+P_{N}}{N}-P_{M}\right\|
$$

and

$$
c\left(M_{1}, \ldots, M_{N}\right)=\frac{N}{N-1}\left\|\frac{P_{1}+\cdots+P_{N}}{N}-P_{M}\right\|-\frac{1}{N-1} .
$$

Proof. We have (see, e.g., [17])

$$
c(\mathbf{C}, \mathbf{D})=\left\|P_{\mathbf{D}} P_{\mathbf{C}}-P_{\mathbf{C} \cap \mathbf{D}}\right\| .
$$

Using Lemma 3.5, $P_{\mathbf{D}} P_{\mathbf{C}}-P_{\mathbf{C} \cap \mathbf{D}}$ can be written as

$$
\frac{1}{N}\left[\begin{array}{cccc}
1 & 1 & \ldots & 1 \\
1 & 1 & \ldots & 1 \\
\vdots & \vdots & & \vdots \\
1 & 1 & \ldots & 1
\end{array}\right]\left[\begin{array}{cccc}
P_{1} & 0 & \ldots & 0 \\
& \ddots & & \\
& & \ddots & \\
0 & 0 & \ldots & P_{N}
\end{array}\right]-\frac{1}{N}\left[\begin{array}{cccc}
P_{M} & P_{M} & \ldots & P_{M} \\
P_{M} & \ldots & \ldots & P_{M} \\
\vdots & & & \vdots \\
P_{M} & P_{M} & \ldots & P_{M}
\end{array}\right]
$$

Therefore,

$$
P_{\mathbf{D}} P_{\mathbf{C}}-P_{\mathbf{C} \cap \mathbf{D}}=\frac{1}{N}\left[\begin{array}{cccc}
P_{1}-P_{M} & P_{2}-P_{M} & \ldots & P_{N}-P_{M} \\
P_{1}-P_{M} & P_{2}-P_{M} & \ldots & P_{N}-P_{M} \\
\vdots & & & \vdots \\
P_{1}-P_{M} & P_{2}-P_{M} & \ldots & P_{N}-P_{M}
\end{array}\right]
$$


whence

$$
\kappa:=\kappa\left(M_{1}, \ldots, M_{N}\right)=c(\mathbf{C}, \mathbf{D})^{2}=\frac{1}{N^{2}}\left\|\left[\begin{array}{cccc}
P_{1}-P_{M} & P_{2}-P_{M} & \ldots & P_{N}-P_{M} \\
P_{1}-P_{M} & P_{2}-P_{M} & \ldots & P_{N}-P_{M} \\
\vdots & & & \vdots \\
P_{1}-P_{M} & P_{2}-P_{M} & \ldots & P_{N}-P_{M}
\end{array}\right]\right\|^{2} .
$$

We see that $N^{2} \kappa$ is equal to

$$
\left\|\left[\begin{array}{cccc}
P_{1}-P_{M} & P_{2}-P_{M} & \ldots & P_{N}-P_{M} \\
P_{1}-P_{M} & P_{2}-P_{M} & \ldots & P_{N}-P_{M} \\
\vdots & & & \vdots \\
P_{1}-P_{M} & P_{2}-P_{M} & \ldots & P_{N}-P_{M}
\end{array}\right]\left[\begin{array}{cccc}
P_{1}-P_{M} & P_{1}-P_{M} & \ldots & P_{1}-P_{M} \\
P_{2}-P_{M} & P_{2}-P_{M} & \ldots & P_{2}-P_{M} \\
\vdots & & & \vdots \\
P_{N}-P_{M} & P_{N}-P_{M} & \ldots & P_{N}-P_{M}
\end{array}\right]\right\|
$$

Therefore,

$$
N^{2} \kappa=\left\|\left[\begin{array}{cccc}
\Sigma & \Sigma & \ldots & \Sigma \\
\Sigma & \Sigma & \ldots & \Sigma \\
\vdots & & & \vdots \\
\Sigma & \Sigma & \ldots & \Sigma
\end{array}\right]\right\|, \quad \text { where } \Sigma:=\sum_{j=1}^{N}\left(P_{j}-P_{M}\right)^{2}
$$

Since

$$
\left(P_{j}-P_{M}\right)^{2}=P_{j}-P_{j} P_{M}-P_{M} P_{j}+P_{M}=\left(I-P_{M}\right) P_{j}
$$

we have

$$
\Sigma=\left(I-P_{M}\right) \sum_{j=1}^{N} P_{j} .
$$

Let $K$ be the matrix all entries of which are equal to $\Sigma$. One way to compute the norm of $K$ is to note that, like every circulant matrix, $K$ is unitarily equivalent to a diagonal matrix. Indeed, denote by $F$ the unitary $N \times N$-matrix representing the discrete Fourier transform $F=N^{-1 / 2}\left[\left(\omega^{j k}\right)\right]_{0 \leq j, k \leq N-1}$, where $\omega=\exp (-2 i \pi / N)$ is a primitive $N$ th root of unity. Then

$$
F^{*} K F=\left[\begin{array}{cccc}
N \Sigma & 0 & \ldots & 0 \\
0 & 0 & \ldots & 0 \\
\vdots & & & \vdots \\
0 & 0 & \ldots & 0
\end{array}\right]
$$

Therefore,

$$
\kappa=\frac{1}{N}\|\Sigma\|=\left\|\left(I-P_{M}\right) \sum_{j=1}^{N} P_{j}\right\|=\left\|\sum_{j=1}^{N} P_{j}\left(I-P_{M}\right)\right\| .
$$

Since $P_{j} P_{M}=P_{M}$, this can be written as

$$
\kappa=\left\|\frac{\sum_{j=1}^{N} P_{j}}{N}-P_{M}\right\| \text {. }
$$

The proof is complete.

The following definition is related to the minimal gap between two subspaces (see 18, p. 219 and Lemma 4.4]). See also the regularity (or bounded linear regularity) condition in [6], and the references therein. 
Definition 3.8. Let $N \geq 2$. Let $M_{1}, \ldots, M_{N}$ be $N$ closed subspaces of $H$ with intersection $M=M_{1} \cap \cdots \cap M_{N}$. The number

$$
\ell\left(M_{1}, \ldots, M_{N}\right)=\inf _{x \notin M} \frac{\max _{1 \leq j \leq N} \operatorname{dist}\left(x, M_{j}\right)}{\operatorname{dist}(x, M)}
$$

is called the inclination of $\left(M_{1}, \ldots, M_{N}\right)$.

Proposition 3.9. Let $N \geq 2$. Let $M_{1}, \ldots, M_{N}$ be $N$ closed subspaces of $H$ with intersection $M=M_{1} \cap \cdots \cap M_{N}$. Then

$$
1-\ell\left(M_{1}, \ldots, M_{N}\right) \leq c(\mathbf{C}, \mathbf{D})=\kappa\left(M_{1}, \ldots, M_{N}\right)^{1 / 2} \leq 1-\frac{\ell\left(M_{1}, \ldots, M_{N}\right)^{2}}{2 N}
$$

and

$$
1-\frac{2 N}{N-1} \ell\left(M_{1}, \ldots, M_{N}\right) \leq c\left(M_{1}, \ldots, M_{N}\right) \leq 1-\frac{\ell\left(M_{1}, \ldots, M_{N}\right)^{2}}{N-1} .
$$

In particular, $\ell\left(M_{1}, \ldots, M_{N}\right)=0$ if and only if $c\left(M_{1}, \ldots, M_{N}\right)=1$ and if and only if $\kappa\left(M_{1}, \ldots, M_{N}\right)=1$.

Proof. Denote $\ell=\ell\left(M_{1}, \ldots, M_{N}\right)$. Let $\varepsilon>0$. There exists $x \in H$ with $\left\|x-P_{M} x\right\|=$ $\operatorname{dist}(x, M)=1 \operatorname{such}$ that $\operatorname{dist}\left(x, M_{j}\right)<\ell+\varepsilon$ for each $j$. Set $u_{j}=P_{j}\left(x-P_{M} x\right)=$ $P_{j} x-P_{M} x$, where $P_{j}$ is the orthogonal projection onto $M_{j}(1 \leq j \leq N)$. Then $u_{j} \in M_{j}$ and $\left\|u_{j}\right\| \leq\left\|x-P_{M} x\right\|=1$. Recall that $\mathbf{C}$ is the $\ell^{2}$-direct sum $\mathbf{C}=M_{1} \times \cdots \times M_{N} \subset H^{N}$, and $\mathbf{D}$ is the diagonal, $\mathbf{D}=\operatorname{diag}(H)=\{(y, \ldots, y): y \in H\} \subset H^{N}$. Observe that $\mathbf{C} \cap \mathbf{D}=\operatorname{diag}(M)$; thus, $\mathbf{y}=\left(y_{1}, \ldots, y_{N}\right) \in \operatorname{diag}(M)^{\perp}$ if and only if $\left\langle y_{1}+\cdots+y_{N}, m\right\rangle=$ 0 for every $m \in M$. Therefore, $\left(y_{1}, \ldots, y_{N}\right) \in \operatorname{diag}(M)^{\perp}$ if and only if $y_{1}+\cdots+y_{N} \in M^{\perp}$.

Consider

$$
\mathbf{d}=\left(\frac{1}{\sqrt{N}}\left(I-P_{M}\right) x, \ldots, \frac{1}{\sqrt{N}}\left(I-P_{M}\right) x\right) \in \mathbf{D} \cap \operatorname{diag}(M)^{\perp}
$$

and

$$
\mathbf{c}=\left(\frac{1}{\sqrt{N}} u_{1}, \ldots, \frac{1}{\sqrt{N}} u_{N}\right) \in \mathbf{C} .
$$

For each $m \in M$, we have

$$
\left\langle\sum_{j=1}^{N} u_{j}, m\right\rangle=\frac{1}{\sqrt{N}}\left[\sum_{j=1}^{N}\left\langle P_{j} x, m\right\rangle-N\left\langle P_{M} x, m\right\rangle\right]=\frac{1}{\sqrt{N}}\left[\sum_{j=1}^{N}\left\langle x, P_{j} m\right\rangle-N\langle x, m\rangle\right]=0 .
$$

Consequently, $\mathbf{c} \in \mathbf{C} \cap \operatorname{diag}(M)^{\perp}$. We also have $\|\mathbf{d}\|=1$ and $\|\mathbf{c}\|^{2}=\frac{1}{N}\left(\left\|u_{1}\right\|^{2}+\cdots+\right.$ $\left.\left\|u_{N}\right\|^{2}\right) \leq 1$. Thus,

$$
c(\mathbf{C}, \mathbf{D}) \geq|\langle\mathbf{c}, \mathbf{d}\rangle|=\frac{1}{N}\left|\left\langle\sum_{j=1}^{N} u_{j}, x-P_{M} x\right\rangle\right| \geq \frac{1}{N} \operatorname{Re}\left\langle\sum_{j=1}^{N} u_{j}, x-P_{M} x\right\rangle .
$$

For a fixed $j$ we have $\left\|x-P_{M} x-u_{j}\right\|=\left\|x-P_{j} x\right\|=\operatorname{dist}\left(x, M_{j}\right)<\ell+\varepsilon$. Therefore,

$$
2 \operatorname{Re}\left\langle x-P_{M} x, u_{j}\right\rangle=\left\|x-P_{M} x\right\|^{2}+\left\|u_{j}\right\|^{2}-\left\|x-P_{M} x-u_{j}\right\|^{2}>1+\left\|u_{j}\right\|^{2}-(\ell+\varepsilon)^{2} .
$$


Also, $\left\|u_{j}\right\| \geq\left\|x-P_{M} x\right\|-\left\|x-P_{M} x-u_{j}\right\|>1-(\ell+\varepsilon)$. We obtain

$$
\begin{aligned}
c(\mathbf{C}, \mathbf{D}) & \geq \frac{1}{N} \operatorname{Re}\left\langle\sum_{j=1}^{N} u_{j}, x-P_{M} x\right\rangle \\
& \geq \frac{1}{2 N} \sum_{j=1}^{N}\left(1+\left\|u_{j}\right\|^{2}-(\ell+\varepsilon)^{2}\right) \\
& \geq \frac{1}{2 N}\left(N+N(1-(\ell+\varepsilon))^{2}-N(\ell+\varepsilon)^{2}\right) \\
& =\frac{1}{2}\left(1+(1-(\ell+\varepsilon))^{2}-(\ell+\varepsilon)^{2}\right) .
\end{aligned}
$$

Since this inequality is true for every $\varepsilon>0$, we get

$$
c(\mathbf{C}, \mathbf{D}) \geq \frac{1-\ell^{2}+(1-\ell)^{2}}{2}=1-\ell .
$$

Denote $c=c(\mathbf{C}, \mathbf{D})$. Let $\varepsilon>0$. There exist $\mathbf{c} \in \mathbf{C} \cap \operatorname{diag}(M)^{\perp}$ and $\mathbf{d} \in \mathbf{D} \cap \operatorname{diag}(M)^{\perp}$ with $\|\mathbf{c}\|=1,\|\mathbf{d}\|=1$ such that $c<|\langle\mathbf{c}, \mathbf{d}\rangle|+\varepsilon$. Let $\theta \in \mathbb{R}$ be such that $\langle\mathbf{c}, \mathbf{d}\rangle=$ $e^{i \theta}|\langle\mathbf{c}, \mathbf{d}\rangle|$. Then

$$
\left\|\mathbf{c}-e^{i \theta} \mathbf{d}\right\|^{2}=2-2 \operatorname{Re}\left(e^{-i \theta}\langle\mathbf{c}, \mathbf{d}\rangle\right)=2-2|\langle\mathbf{c}, \mathbf{d}\rangle| \leq 2-2(c-\varepsilon) .
$$

Set $\mathbf{c}=\left(m_{1}, \ldots, m_{N}\right) \in \mathbf{C} \cap \operatorname{diag}(M)^{\perp}$ and $e^{i \theta} \mathbf{d}=(y, \ldots, y) \in \mathbf{D} \cap \operatorname{diag}(M)^{\perp}$. Then $y \in M^{\perp}$ and

$$
\left\|m_{1}\right\|^{2}+\cdots+\left\|m_{N}\right\|^{2}=1, \quad\|y\|=1 / \sqrt{N}, \quad \text { and } \quad \sum_{j=1}^{N}\left\|m_{j}-y\right\|^{2} \leq 2-2(c-\varepsilon) .
$$

Let $x=\sqrt{N} y$. Then $x \in M^{\perp}, \operatorname{dist}(x, M)=\|x\|=1$, and

$$
\operatorname{dist}\left(x, M_{j}\right)^{2} \leq\left\|x-\sqrt{N} m_{j}\right\|^{2}=N\left\|m_{j}-y\right\|^{2} \leq N(2-2(c-\varepsilon)) .
$$

Finally, we obtain $\ell^{2} \leq 2 N(1-c)$, whence

$$
1-\ell \leq c(\mathbf{C}, \mathbf{D}) \leq 1-\frac{\ell^{2}}{2 N} .
$$

Using the identities

$$
c(\mathbf{C}, \mathbf{D})=\kappa\left(M_{1}, \ldots, M_{N}\right)^{1 / 2}
$$

and

$$
\kappa\left(M_{1}, \ldots, M_{N}\right)=\frac{N-1}{N} c\left(M_{1}, \ldots, M_{N}\right)+\frac{1}{N},
$$

we obtain

$$
\frac{N(1-\ell)^{2}-1}{N-1} \leq c\left(M_{1}, \ldots, M_{N}\right) \leq \frac{N\left(1-\left(\ell^{2} / 2 N\right)^{2}\right)-1}{N-1} .
$$

Since $2-\ell \leq 2$, we can write

$$
\frac{N(1-\ell)^{2}-1}{N-1}=1-\frac{N}{N-1} \ell(2-\ell) \geq 1-\frac{2 N}{N-1} \ell .
$$

Also, we have

$$
\frac{N\left(1-\left(\ell^{2} / 2 N\right)^{2}\right)-1}{N-1}=1-\frac{\ell^{2}}{N-1}\left(1-\frac{\ell^{2}}{4 N}\right) \leq 1-\frac{\ell^{2}}{N-1} .
$$




\section{$\S 4$. Characterizing (ASC) For products of projections}

4A. A qualitative result. When $T$ is the product of $N$ orthogonal projections, we know from Theorem 2.4 that the dichotomy (QUC)/(ASC) holds, and that we have quick uniform convergence if and only if the range of $T-I$ is closed. The following qualitative result gives a characterization of the (ASC) condition in terms of several parameters associated with $\left(M_{1}, \ldots, M_{N}\right)$, or spectral properties of $T$, or random products. We denote by $\|\cdot\|_{e}$ the essential norm and by $\sigma_{e}$ the essential spectrum.

Theorem 4.1. Let $N \geq 2$. Let $M_{1}, \ldots, M_{N}$ be $N$ closed subspaces of $H$ with intersection $M=M_{1} \cap \cdots \cap M_{N}$. Denote by $P_{j}$ the orthogonal projection onto $M_{j}, 1 \leq j \leq N$, and by $P_{M}$ the orthogonal projection onto $M$. Put $T=P_{N} P_{N-1} \ldots P_{1}$. The following assertions are equivalent:

(1) $\operatorname{Ran}(T-I)$ is not closed;

( $\left.1^{\prime}\right)$ for every $k \geq N$ and every sequence of indices $\left(i_{j}\right)_{j \geq 1}$ such that $\left\{i_{1}, \ldots, i_{k}\right\}=$ $\{1,2, \ldots, N\}, \operatorname{Ran}\left(P_{i_{k}} \ldots P_{i_{1}}-I\right)$ is not closed;

(2) one of the conditions (ASC1), (ASC2), (ASC3), and (ASCH) is fulfilled for $T$;

$\left(2^{\prime}\right)(\mathrm{ASCH})$ for random products: for every $\varepsilon>0$, every sequence $\left(a_{n}\right)_{n \geq 0}$ of positive reals with $\lim _{n \rightarrow \infty} a_{n}=0$, and every sequence of indices $\left(i_{k}\right)_{k \geq 1}$ in $\{1,2, \ldots, N\}$, there exists $x \in H$ with $\|x\|<\sup _{n} a_{n}+\varepsilon$ such that

$$
\operatorname{Re}\left\langle P_{i_{n}} P_{i_{n-1}} \ldots P_{i_{1}} x-P_{M} x, x\right\rangle>a_{n}
$$

for each $n \geq 1$;

(3) $c\left(M_{1}, \ldots, M_{N}\right)=1$, or, equivalently, $\kappa\left(M_{1}, \ldots, M_{N}\right)=1$, or $\ell\left(M_{1}, \ldots, M_{N}\right)=$ 0

(4) for every $\varepsilon>0$, every closed subspace $K \subset M^{\perp}$ of finite codimension (in $M^{\perp}$ ), there exists $x \in K$ such that $\|x\|=1$ and $\max \left\{\operatorname{dist}\left(x, M_{j}\right): j=1, \ldots, N\right\}<\varepsilon$;

(5) $1 \in \sigma\left(T-P_{M}\right)$;

$\left(5^{\prime}\right)$ for every $k$ and every $i_{1}, \ldots, i_{k} \in\{1,2, \ldots, N\}$, we have $1 \in \sigma\left(P_{i_{k}} \ldots P_{i_{1}}-P_{M}\right)$;

(6) $\left\|T-P_{M}\right\|=1$;

$\left(6^{\prime}\right)$ for every $k$ and every sequence of indices $\left(i_{k}\right)_{k \geq 1}, 1 \leq i_{k} \leq N$, with $\left\{i_{1}, \ldots, i_{k}\right\}=$ $\{1,2, \ldots, N\}$, we have $\left\|P_{i_{k}} \ldots P_{i_{1}}-P_{M}\right\|=1$

(7) $\left\|T-P_{M}\right\|_{e}=1$;

$\left(7^{\prime}\right)$ for every $k$ and every $i_{1}, \ldots, i_{k} \in\{1,2, \ldots, N\}$, we have $\left\|P_{i_{k}} \ldots P_{i_{1}}-P_{M}\right\|_{e}=1$;

(8) $1 \in \sigma_{e}\left(T-P_{M}\right)$;

(8') for every $k$ and every $i_{1}, \ldots, i_{k} \in\{1,2, \ldots, N\}$, we have $1 \in \sigma_{e}\left(P_{i_{k}} \ldots P_{i_{1}}-P_{M}\right)$;

(9) for every $\varepsilon>0$ and every closed subspace $K \subset M^{\perp}$ of finite codimension (in $\left.M^{\perp}\right)$, there exists $x \in K$ such that $\|T x-x\| \leq \varepsilon$;

$\left(9^{\prime}\right)$ for every $\varepsilon>0$ and every closed subspace $K \subset M^{\perp}$ of finite codimension (in $\left.M^{\perp}\right)$, there exists $x \in K$ such that $\left\|P_{i_{k}} \ldots P_{i_{1}} x-x\right\| \leq \varepsilon$ for every $k$ and every $i_{1}, \ldots, i_{k} \in\{1,2, \ldots, N\}$

(10) the sum of $\operatorname{diag}\left(M_{1}\right) \subset H^{N-1}$ and $M_{2} \oplus \cdots \oplus M_{N} \subset H^{N-1}$ is not closed in $H^{N-1}$ (and equivalent statements for $\operatorname{diag}\left(M_{j}\right) \subset H^{N-1}, 2 \leq j \leq N$ );

(11) $M_{1}^{\perp}+\cdots+M_{N}^{\perp}$ is not closed in $H$.

Conditions (1), (2), (5), (6), (7), (8), and (9), most of which are of spectral nature, are conditions about $T=P_{N} \ldots P_{1}$, while the corresponding conditions denoted with primes are similar conditions about random products $P_{i_{N}} \ldots P_{i_{1}}$. Conditions $(3),(4),(10)$, and (11) are about the geometry of the subspaces $M_{j}$.

Notice that we have the dichotomy (QUC)/(ASC) in all possible senses, and that (QUC) holds if and only if $c\left(M_{1}, \ldots, M_{N}\right)<1$. A quantitative estimate reflecting the 
geometric convergence of $\left\|T^{n}-P_{M}\right\|$ to zero, in terms of the Friedrichs number, will be given after the proof of the theorem.

Proof of Theorem 4.1. “(1) $\Leftrightarrow(2)$ " The equivalence of (1) and (2) follows from Theorem 2.4 .

"(1) $\Leftrightarrow(5)$ " The equivalence of (1) and (5) follows from the proof of Theorem 2.1 (see also Remark 2.2). Observe that, with respect to the decomposition $H=M \oplus M^{\perp}$, we have $T=P_{M} \oplus A$, where $A=\left.T\right|_{M^{\perp}}=T\left(I-P_{M}\right)=T-P_{M}$.

"(1) $\Rightarrow(3)$ " We prove this implication in a quantitative form. We denote by

$$
\gamma=\gamma(I-T)=\inf \{\|x-T x\|: x \in H, \operatorname{dist}(x, \operatorname{Ker}(T-I)=1\}
$$

the reduced minimum modulus of $T-I$. Then $\operatorname{Ran}(T-I)$ is closed if and only if $\gamma>0$. Clearly, $T y=y$ for $y \in M$. If $T x=x$, then

$$
\|x\|=\left\|P_{N} \ldots P_{1} x\right\| \leq\left\|P_{N-1} \ldots P_{1} x\right\| \leq \cdots \leq\left\|P_{1} x\right\| \leq\|x\| .
$$

We successively obtain $P_{1} x=x, P_{2} x=x, \ldots, P_{N} x=x$, and finally, $x \in M$. Thus, $\operatorname{Ker}(T-I)=M$.

Let $\varepsilon>0$. There exists $x \in H$ with $\left\|x-P_{M} x\right\|=\operatorname{dist}(x, M)=1$ such that $\|x-T x\| \leq$ $\gamma+\varepsilon$. We obtain

$$
\begin{aligned}
1=\left\|x-P_{M} x\right\| & \geq\left\|P_{1}\left(x-P_{M} x\right)\right\|=\left\|P_{1} x-P_{M} x\right\| \geq\left\|P_{2} P_{1} x-P_{M} x\right\| \\
& \geq \cdots \geq\left\|P_{N} \ldots P_{1} x-P_{M} x\right\|=\left\|T x-P_{M} x\right\| \\
& \geq\left\|x-P_{M} x\right\|-\|x-T x\| \geq 1-\gamma-\varepsilon .
\end{aligned}
$$

We also have

$$
\begin{aligned}
\left\|\left(I-P_{1}\right)\left(x-P_{M} x\right)\right\|^{2} & =\left\|x-P_{M} x\right\|^{2}-\left\|P_{1} x-P_{M} x\right\|^{2} \\
& \leq 1-(1-\gamma-\varepsilon)^{2}=-(\gamma+\varepsilon)^{2}+2(\gamma+\varepsilon) \leq 2 \gamma+2 \varepsilon .
\end{aligned}
$$

Thus, $\operatorname{dist}\left(x, M_{1}\right)=\left\|x-P_{1} x\right\|=\left\|\left(I-P_{1}\right)\left(x-P_{M} x\right)\right\| \leq(2 \gamma+2 \varepsilon)^{1 / 2}$.

Let $y=x-P_{M} x$; then $\|y\|=1$. For a fixed $s$ between 1 and $N$, we can write

$$
\begin{aligned}
\left\|P_{s} \ldots P_{1} y-P_{s+1} \ldots P_{1} y\right\|^{2} & =\left\|P_{s} \ldots P_{1} y\right\|^{2}-\left\|P_{s+1} \ldots P_{1} y\right\|^{2} \\
& \leq\|y\|^{2}-\left\|P_{s+1} \ldots P_{1} x-P_{M} x\right\|^{2} \\
& \leq 1-(1-\gamma-\varepsilon)^{2} \leq 2 \gamma+2 \varepsilon .
\end{aligned}
$$

Consequently,

$$
\begin{aligned}
\operatorname{dist}\left(x, M_{j}\right) & =\operatorname{dist}\left(y, M_{j}\right) \leq\left\|y-P_{j} \ldots P_{1} y\right\| \\
& \leq\left\|y-P_{1} y\right\|+\left\|P_{1} y-P_{2} P_{1} y\right\|+\cdots+\left\|P_{j-1} \ldots P_{1} y-P_{j} \ldots P_{1} y\right\| \\
& \leq j \sqrt{(2 \gamma+2 \varepsilon)}
\end{aligned}
$$

for every $j$. Hence, $\max _{1 \leq j \leq N} \operatorname{dist}\left(x, M_{j}\right) \leq N \sqrt{(2 \gamma+2 \varepsilon)}$ and, since $\varepsilon$ is arbitrary,

$$
\ell:=\ell\left(M_{1}, \ldots, M_{N}\right) \leq N \sqrt{2 \gamma}
$$

We obtain $\frac{1}{2 N^{2}} \ell\left(M_{1}, \ldots, M_{N}\right)^{2} \leq \gamma(T-I)$. Therefore, if $\operatorname{Ran}(I-T)$ is not closed $(\gamma=0)$, then $\ell\left(M_{1}, \ldots, M_{N}\right)=0$.

"(3) $\Rightarrow\left(1^{\prime}\right)$ " Let $k \geq N$, and let $\left(i_{j}\right)_{j \geq 1}$ be a sequence of indices with $\left\{i_{1}, \ldots, i_{k}\right\}=$ $\{1,2, \ldots, N\}$. This implies that $\operatorname{Ker}\left(I-P_{i_{k}} P_{i_{k-1}} \ldots P_{i_{1}}\right)=M$. Let $\ell=\ell\left(M_{1}, \ldots, M_{N}\right)$, and let $\varepsilon>0$. There exists $x \in H$ with $\left\|x-P_{M} x\right\|=\operatorname{dist}(x, M)=1$ such that $\max _{j} \operatorname{dist}\left(x, M_{j}\right)<\ell+\varepsilon$. We have

$$
\left\|x-P_{i_{1}} x\right\|=\operatorname{dist}\left(x, M_{i_{1}}\right)<\ell+\varepsilon
$$


and

$$
\left\|P_{i_{2}} P_{i_{1}} x-P_{i_{1}} x\right\|=\operatorname{dist}\left(P_{i_{1}} x, M_{i_{2}}\right) \leq\left\|x-P_{i_{1}} x\right\|+\operatorname{dist}\left(x, M_{i_{2}}\right)<2(\ell+\varepsilon) .
$$

Set $x_{0}=x$ and $x_{s}=P_{i_{s}} P_{i_{s-1}} \ldots P_{i_{1}} x$ for $s \geq 1$. Suppose that

$$
\left\|x_{s}-x_{s-1}\right\| \leq 2^{s-1}(\ell+\varepsilon)
$$

for $1 \leq s \leq r$. Then

$$
\begin{aligned}
\left\|x_{r+1}-x_{r}\right\| & =\operatorname{dist}\left(P_{i_{r}} \ldots P_{i_{1}} x, M_{i_{r+1}}\right) \\
& \leq\left\|P_{i_{r}} \ldots P_{i_{1}} x-x\right\|+\operatorname{dist}\left(x, M_{i_{r+1}}\right) \\
& \leq\left\|x_{s}-x_{s-1}\right\|+\left\|x_{s-1}-x_{s-2}\right\|+\cdots+\left\|x_{1}-x\right\|+\operatorname{dist}\left(x, M_{r+1}\right) \\
& \leq\left(2^{r-1}+2^{r-2}+\cdots+2+1+1\right)(\ell+\varepsilon)=2^{r}(\ell+\varepsilon) .
\end{aligned}
$$

Therefore, (4.1) is true for every $s$, and we obtain

$$
\begin{aligned}
\left\|P_{i_{k}} P_{i_{k-1}} \ldots P_{i_{1}} x-x\right\| & =\left\|x_{k}-x\right\| \\
& \leq\left\|x_{k}-x_{k-1}\right\|+\left\|x_{k-1}-x_{k-2}\right\|+\cdots+\left\|x_{1}-x\right\| \\
& \leq\left(2^{k-1}+2^{k-2}+\cdots+1\right)(\ell+\varepsilon)=\left(2^{k}-1\right)(\ell+\varepsilon) .
\end{aligned}
$$

Thus, $\gamma\left(P_{i_{k}} P_{i_{k-1}} \ldots P_{i_{1}}-I\right) \leq\left(2^{k}-1\right)(\ell+\varepsilon)$. Letting $\varepsilon \rightarrow 0$, we obtain

$$
\gamma\left(P_{i_{k}} P_{i_{k-1}} \ldots P_{i_{1}}-I\right) \leq\left(2^{k}-1\right) \ell
$$

This shows that if $\ell=0$, or equivalently, if $c\left(M_{1}, \ldots, M_{N}\right)=1$, then the range of $P_{i_{k}} P_{i_{k-1}} \ldots P_{i_{1}}-I$ is not closed.

The implication " $\left(1^{\prime}\right) \Rightarrow(1)$ " is clear. Note also that the above proof for $k=N$ and $i_{s}=s$ implies that

$$
\frac{1}{2 N^{2}} \ell^{2} \leq \gamma(T-I) \leq\left(2^{N}-1\right) \ell
$$

Here $\ell=\ell\left(M_{1}, \ldots, M_{N}\right)$.

" $\left(1^{\prime}\right) \Rightarrow\left(6^{\prime}\right)$ " Note that we always have $\left\|P_{i_{k}} \ldots P_{i_{1}}-P_{M}\right\| \leq 1$. Now, suppose that $a:=\left\|P_{i_{k}} \ldots P_{i_{1}}-P_{M}\right\|<1$. We want to show that the range of $I-P_{i_{k}} \ldots P_{i_{1}}$ is closed. First, observe that $\operatorname{Ker}\left(I-P_{i_{k}} \ldots P_{i_{1}}\right)=M$ because $\left\{i_{1}, \ldots, i_{k}\right\}=\{1,2, \ldots, N\}$. Let $x \in H$ be such that $\operatorname{dist}(x, M)=\left\|x-P_{M} x\right\|=1$. We have

$$
\begin{aligned}
\left\|\left(I-P_{i_{k}} \ldots P_{i_{1}}\right) x\right\| & =\left\|x-P_{M} x+P_{M} x-P_{i_{k}} \ldots P_{i_{1}} x\right\| \\
& \geq 1-\left\|P_{i_{k}} \ldots P_{i_{1}} x-P_{M} x\right\| \\
& =1-\left\|\left(P_{i_{k}} \ldots P_{i_{1}}-P_{M}\right)\left(x-P_{M} x\right)\right\| \\
& \geq 1-a .
\end{aligned}
$$

Therefore, the reduced minimum modulus of $I-P_{i_{k}} \ldots P_{i_{1}}$ satisfies $\gamma\left(I-P_{i_{k}} \ldots P_{i_{1}}\right) \geq$ $1-\left\|P_{i_{k}} \ldots P_{i_{1}}-P_{M}\right\|$. In particular, $\operatorname{Ran}\left(I-P_{i_{k}} \ldots P_{i_{1}}\right)$ is closed if $a<1$.

The implication " $\left(6^{\prime}\right) \Rightarrow(6)$ " is easy.

Lemma 4.2. Let $x \in H$, and set $u_{j}=P_{j} \ldots P_{1} x-P_{M} x$ for $j \geq 1, u_{0}=x-P_{M} x$. For every $j$ with $1 \leq j \leq N$, we have

$$
\left\|u_{j-1}-u_{j}\right\|^{2} \leq\left\|u_{j-1}\right\|^{2}-\left\|T x-P_{M} x\right\|^{2} \leq\left\|x-P_{M} x\right\|^{2}-\left\|T x-P_{M} x\right\|^{2} .
$$

Proof. We can write

$$
\left\|T x-P_{M} x\right\|=\left\|u_{N}\right\|=\left\|P_{N} u_{N-1}\right\| \leq\left\|u_{N-1}\right\| \leq \cdots \leq\left\|u_{0}\right\|=\left\|x-P_{M} x\right\| .
$$


Therefore,

$$
\begin{aligned}
\left\|u_{j-1}-u_{j}\right\|^{2}+\left\|T x-P_{M} x\right\|^{2} & =\left\|u_{j-1}-P_{j} u_{j-1}\right\|^{2}+\left\|P_{N} \ldots P_{j+1} P_{j} u_{j-1}\right\|^{2} \\
& \leq\left\|u_{j-1}-P_{j} u_{j-1}\right\|^{2}+\left\|P_{j} u_{j-1}\right\|^{2} \\
& =\left\|u_{j-1}\right\|^{2} \\
& =\left\|P_{j-1} \ldots P_{1}\left(x-P_{M} x\right)\right\|^{2} \\
& \leq\left\|x-P_{M} x\right\|^{2},
\end{aligned}
$$

completing the proof of the lemma.

We continue the proof of Theorem 4.1

"(6) $\Rightarrow(3)$ " Let $j$ be between 1 and $N$. Using the Cauchy-Schwarz inequality and Lemma 4.2, we obtain

$$
\begin{aligned}
\operatorname{dist}\left(x, M_{j}\right)^{2} & \leq\left\|x-P_{j} \ldots P_{1} x\right\|^{2} \\
& \leq\left(\left\|x-P_{1} x\right\|+\left\|P_{1} x-P_{2} P_{1} x\right\|+\cdots+\left\|P_{j-1} \ldots P_{1} x-P_{j} \ldots P_{1} x\right\|\right)^{2} \\
& \leq j\left(\left\|u_{0}-u_{1}\right\|^{2}+\left\|u_{1}-u_{2}\right\|^{2}+\cdots+\left\|u_{j-1}-u_{j}\right\|^{2}\right) \\
& \leq j^{2}\left(\left\|x-P_{M} x\right\|^{2}-\left\|T x-P_{M} x\right\|^{2}\right) \\
& \leq N^{2}\left(\left\|x-P_{M} x\right\|^{2}-\left\|T x-P_{M} x\right\|^{2}\right) .
\end{aligned}
$$

We get

$$
N^{2}\left(\left\|x-P_{M} x\right\|^{2}-\left\|T x-P_{M} x\right\|^{2}\right) \geq \max _{1 \leq j \leq N} \operatorname{dist}\left(x, M_{j}\right)^{2} \geq \ell^{2}\left\|x-P_{M} x\right\|^{2},
$$

which yields

In particular,

$$
\left\|T x-P_{M} x\right\|^{2} \leq\left(1-\frac{\ell^{2}}{N^{2}}\right)\left\|x-P_{M} x\right\|^{2}
$$

$$
\left\|T-P_{M}\right\| \leq \sqrt{1-\frac{\ell^{2}}{N^{2}}}
$$

Therefore, $\left\|T-P_{M}\right\|=1$ implies $\ell=0$, i.e., (6) implies (3).

"(1) $\Rightarrow(9)$ " Let $\varepsilon>0$. Let $K \subset M^{\perp}$ be a closed subspace of finite codimension in $M^{\perp}$. With respect to the decomposition $H=M \oplus M^{\perp}$, the operator $T$ has the following matrix decomposition:

$$
T=\left(\begin{array}{cc}
I & 0 \\
0 & A
\end{array}\right)
$$

Since $\operatorname{Ran}(T-I)$ is not closed, the range of the operator $I-A$, acting on $M^{\perp}$, is not closed. This means that $I-A \in \mathcal{B}\left(M^{\perp}\right)$ is not an upper semi-Fredholm operator, and therefore, there exists $x \in K$ such that $\|x\|=1$ and $\|x-A x\| \leq \varepsilon$. It follows that $\|x-T x\| \leq \varepsilon$.

"(9) $\Rightarrow(4)$ " Let $x$ be as in (9). Then $x \in K,\|x\|=1$, and $\|x-T x\| \leq \varepsilon$. We have

$$
1=\|x\| \geq\left\|P_{1} x\right\| \geq\left\|P_{2} P_{1} x\right\| \geq \cdots \geq\|T x\| \geq 1-\varepsilon .
$$

Set $x_{s}=P_{s} P_{s-1} \ldots P_{1} x$ for $s \geq 1$ and $x_{0}=x$. Then $x_{s} \in M_{s} \cap M^{\perp}$ for each $s \geq 0$ and $x_{s-1}-x_{s}=\left(I-P_{s}\right) x_{s-1}$ is orthogonal to $x_{s}$. Hence,

$$
\left\|x_{s-1}-x_{s}\right\|^{2}=\left\|x_{s-1}\right\|^{2}-\left\|x_{s}\right\|^{2} \leq 1-(1-\varepsilon)^{2}<2 \varepsilon
$$

and $\left\|x_{s-1}-x_{s}\right\| \leq \sqrt{2 \varepsilon}$ for each $s$. We obtain

$$
\operatorname{dist}\left(x, M_{1}\right)=\left\|x-P_{1} x\right\|=\left\|x_{0}-x_{1}\right\| \leq \sqrt{2 \varepsilon} .
$$


For $s \geq 1$, we have $\operatorname{dist}\left(x, M_{s}\right) \leq\left\|x-P_{s} P_{s-1} \ldots P_{1} x\right\|$; hence,

$$
\begin{aligned}
\operatorname{dist}\left(x, M_{s}\right) & \leq\left\|x-P_{1} x\right\|+\left\|P_{1} x-P_{2} P_{1} x\right\|+\cdots+\left\|P_{s-1} \ldots P_{1} x-P_{s} P_{s-1} \ldots P_{1} x\right\| \\
& \leq s \sqrt{2 \varepsilon} .
\end{aligned}
$$

Therefore, $\max \left\{\operatorname{dist}\left(x, M_{j}\right): j=1, \ldots, N\right\} \leq N \sqrt{2 \varepsilon}$. Since $\varepsilon>0$ is arbitrary, the proof of this implication is over.

"(4) $\Rightarrow\left(9^{\prime}\right)$ " Suppose that (4) is true. Let $\varepsilon>0$, and let $K \subset M^{\perp}$ be a closed subspace of finite codimension in $M^{\perp}$. Then there exists $x \in K$ such that $\operatorname{dist}(x, M)=\|x\|=1$ and $\max \left\{\operatorname{dist}\left(x, M_{j}\right): j=1, \ldots, N\right\} \leq \varepsilon$. Let $i_{1}, \ldots, i_{k} \in\{1,2, \ldots, N\}$. Set $x_{0}=x$, $x_{s}=P_{i_{s}} \ldots P_{i_{1}} x$ for $s \geq 1$. Then $x_{0} \in K$ and $x_{s} \in M^{\perp} \cap M_{i_{s}}$ for $s \geq 1$.

We shall use induction to prove the following two claims:

$$
\operatorname{dist}\left(x_{s}, M_{j}\right) \leq 2^{s} \varepsilon \quad(j \geq 1)
$$

and

$$
\left\|x_{s}-x_{0}\right\| \leq\left(2^{s}-1\right) \varepsilon \quad(s \geq 1) .
$$

Both claims are clearly true for $s=0$. Suppose that both inequalities are true for some $s \geq 0$. Then, using the induction hypothesis several times, we obtain

$$
\begin{aligned}
\left\|x_{s+1}-x_{0}\right\| & \leq\left\|x_{s+1}-x_{s}\right\|+\left\|x_{s}-x_{0}\right\| \\
& =\operatorname{dist}\left(x_{s}, M_{i_{s+1}}\right)+\left\|x_{s}-x_{0}\right\| \leq 2^{s} \varepsilon+\left(2^{s}-1\right) \varepsilon=\left(2^{s+1}-1\right) \varepsilon .
\end{aligned}
$$

For $j \geq 1$ we can write

$$
\operatorname{dist}\left(x_{s+1}, M_{j}\right) \leq\left\|x_{s+1}-x_{0}\right\|+\operatorname{dist}\left(x_{0}, M_{j}\right) \leq\left(2^{s+1}-1\right) \varepsilon+\varepsilon=2^{s+1} \varepsilon .
$$

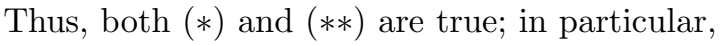

$$
\left\|P_{i_{k}} \ldots P_{i_{1}} x-x\right\|=\left\|x_{k}-x_{0}\right\| \leq 2^{k} \varepsilon .
$$

Since $\varepsilon>0$ was arbitrary, we obtain $\left(9^{\prime}\right)$.

" $\left(9^{\prime}\right) \Rightarrow\left(8^{\prime}\right)$ " We have $P_{s} P_{s-1} \ldots P_{1}-P_{M}=P_{s} P_{s-1} \ldots P_{1}\left(I-P_{M}\right)$, so that the range of this operator is in $M^{\perp}$. Assertion $\left(9^{\prime}\right)$ implies that 1 belongs to the essential spectrum of the restriction of $P_{s} P_{s-1} \ldots P_{1}-P_{M}$ to $M^{\perp}$. Therefore, $1 \in \sigma_{e}\left(P_{s} P_{s-1} \ldots P_{1}-P_{M}\right)$.

The implication " $\left(8^{\prime}\right) \Rightarrow(8)$ " is clear.

"(8) $\Rightarrow(7) \Rightarrow(6)$ " Statement (8) implies the following sequence of inequalities for the essential spectral radius $r_{e}\left(T-P_{M}\right)$ and the essential norm of $T-P_{M}$ :

$$
1 \leq r_{e}\left(T-P_{M}\right) \leq\left\|T-P_{M}\right\|_{e} \leq\left\|T-P_{M}\right\|=\left\|P_{N} \ldots P_{1}\left(I-P_{M}\right)\right\| \leq 1 .
$$

Thus, all these inequalities are equalities.

The proofs of the implications " $\left(8^{\prime}\right) \Rightarrow\left(7^{\prime}\right) \Rightarrow\left(6^{\prime}\right)$ " are similar. The implications " $\left(8^{\prime}\right)$ $\Rightarrow\left(5^{\prime}\right) \Rightarrow(5) "$ are clear.

" $\left(9^{\prime}\right) \Rightarrow\left(2^{\prime}\right)$ " Let $A_{k}$ be the operator $P_{i_{k}} P_{i_{k-1}} \ldots P_{i_{1}}-P_{M}$ restricted to $M^{\perp}$. Condition $\left(9^{\prime}\right)$ implies that 1 is in the boundary of the essential spectrum of the operator $A_{k}$. In accordance with [1, on the space $M^{\perp}$ the operators $A_{k}$ converge weakly to 0 . Assertion $\left(2^{\prime}\right)$ can be proved exactly as in [4, Theorem 1] by replacing there $T^{n}$ by $A_{n}$.

The implication " $\left(2^{\prime}\right) \Rightarrow(2)$ " is clear.

"(10) $\Leftrightarrow(3) "$ We have $\ell\left(M_{1}, \ldots, M_{N}\right)=0$ if and only if $c\left(\operatorname{diag}\left(M_{1}\right), M_{2} \oplus \cdots \oplus M_{N}\right)=$ 1. The proof of this assertion is similar to that of the first part of Proposition 3.9. Therefore, $\ell\left(M_{1}, \ldots, M_{N}\right)=0$, or equivalently, $c\left(M_{1}, \ldots, M_{N}\right)=1$, if and only if $\operatorname{diag}\left(M_{1}\right)+M_{2} \oplus \cdots \oplus M_{N}$ is not closed in $H^{N-1}$.

The implication "(11) $\Leftrightarrow(3)$ " follows from [7]. The proof is complete. 
4B. Quantitative statements. Some remarks concerning the proof of Theorem 4.1 are in order.

Remark 4.3. The proof of Theorem 4.1 gives some quantitative information about the parameters involved. Some other estimates can be proved in a similar way. For instance, here we present the quantitative version of the implication " $\left(6^{\prime}\right) \Rightarrow(3)$ ". Let $k \geq 1$. Suppose $i_{1}, \ldots, i_{k} \in\{1,2, \ldots, N\}$ and $\left\{i_{1}, \ldots, i_{k}\right\}=\{1,2, \ldots, N\}$. Denote $\ell=\ell\left(M_{1}, \ldots, M_{N}\right)$ and

$$
a:=\left\|P_{i_{k}} \ldots P_{i_{1}}-P_{M}\right\| \leq 1 .
$$

Let $\varepsilon>0$. There exists $x \in H$ with $\|x\|=1$ such that $\left\|P_{i_{k}} \ldots P_{i_{1}} x-P_{M} x\right\|>a-\varepsilon$. Denote $y=x-P_{M} x$ and

$$
x_{s}=P_{i_{s}} \ldots P_{i_{1}} x-P_{M} x, \quad x_{0}=x-P_{M} x=y \quad(s \geq 1) .
$$

Clearly,

$$
1=\|x\|=\sqrt{\|y\|^{2}+\left\|P_{M} x\right\|^{2}} \geq\|y\|=\left\|x_{0}\right\| \geq\left\|x_{1}\right\| \geq \cdots \geq\left\|x_{k}\right\|>a-\varepsilon .
$$

Since $x_{s-1}-x_{s}=\left(I-P_{i_{s}}\right) x_{s-1}$ is orthogonal to $M_{i_{s}}$, and $x_{s} \in M_{i_{s}}$, we have

$$
\left\|x_{s-1}-x_{s}\right\|^{2}=\left\|x_{s-1}\right\|^{2}-\left\|x_{s}\right\|^{2} \leq\|y\|^{2}-(a-\varepsilon)^{2} .
$$

For each $r \in\{1, \ldots, k\}$ we can write

$$
\begin{aligned}
\left\|x-P_{M} x-x_{r}\right\| & \leq\left\|x_{0}-x_{1}\right\|+\left\|x_{1}-x_{2}\right\|+\cdots+\left\|x_{r-1}-x_{r}\right\| \\
& \leq k \sqrt{\|y\|^{2}-(a-\varepsilon)^{2}} .
\end{aligned}
$$

Since $\left\{i_{1}, \ldots, i_{k}\right\}=\{1,2, \ldots, N\}$, for each $j \in\{1,2, \ldots, N\}$ we have $\left\{x_{1}, \ldots, x_{k}\right\} \cap M_{j} \neq$ $\varnothing$. Therefore,

$$
\operatorname{dist}\left(x, M_{j}\right) \leq \max \left\{\left\|x-P_{M} x-x_{r}\right\|: 1 \leq r \leq k\right\} \leq k \sqrt{\|y\|^{2}-(a-\varepsilon)^{2}}
$$

and

$$
\|y\|^{2} \ell^{2} \leq \max \left\{\operatorname{dist}\left(x, M_{j}\right)^{2}: 1 \leq j \leq k\right\} \leq k^{2}\left(\|y\|^{2}-(a-\varepsilon)^{2}\right) .
$$

Hence, $k^{2}(a-\varepsilon)^{2} \leq\left(k^{2}-\ell^{2}\right)\|y\|^{2} \leq\left(k^{2}-\ell^{2}\right)$. Since this is true for every $\varepsilon>0$, we obtain $\left\|P_{i_{k}} \ldots P_{i_{1}}-P_{M}\right\|=a \leq\left(1-\ell^{2} / k^{2}\right)^{1 / 2}$.

Theorem 4.4. Let $N \geq 2$. Let $H$ be a complex Hilbert space. Let $M_{1}, \ldots, M_{N}$ be $N$ closed subspaces of $H$ with intersection $M=M_{1} \cap \cdots \cap M_{N}$. Let $P_{j}=P_{M_{j}}, 1 \leq j \leq N$, and let $P_{M}$ be the corresponding orthogonal projections. Denote $T=P_{N} \ldots P_{1}$.

(i) Suppose that $c:=c\left(M_{1}, \ldots, M_{N}\right)<1$. Then $\left(T^{n}\right)_{n \geq 1}$ is uniformly convergent to $P_{M}$, and

$$
\left\|T^{n}-P_{M}\right\| \leq\left(1-\left(\frac{1-c}{4 N}\right)^{2}\right)^{n / 2} \quad(n \geq 1) .
$$

(ii) Suppose that $c:=c\left(M_{1}, \ldots, M_{N}\right)=1$. Then $\left(T^{n}\right)_{n \geq 1}$ is strongly convergent to $P_{M}$ and we have $(A S C)$, in all possible meanings of this paper.

Proof. We only need to prove the estimate in Part (i). Assume $c:=c\left(M_{1}, \ldots, M_{N}\right)<1$. Denote $\widetilde{M}_{j}=M_{j} \cap M^{\perp}$ and $Q_{j}=P_{\widetilde{M}_{j}}$ for $1 \leq j \leq N$. Then the intersection of $\widetilde{M}_{j}$, $1 \leq j \leq N$, is $\{0\}$ and, by Proposition 3.6 (b), we have $c\left(\widetilde{M}_{1}, \ldots, \widetilde{M}_{N}\right)=c<1$. We also have $T^{n}-P_{M}=\left(Q_{N} Q_{N-1} \ldots Q_{1}\right)^{n}$ for each $n \geq 1$ (see [10, Lemma 9.30]). We apply inequality (4.3), which was proved in the implication " $(6) \Rightarrow(3)$ " of Theorem 4.1. to $Q_{j}$. We obtain $\left\|Q_{N} Q_{N-1} \ldots Q_{1}\right\| \leq \sqrt{1-\frac{\ell^{2}}{N^{2}}}$, where now $\ell=\ell\left(\widetilde{M}_{1}, \ldots, \widetilde{M}_{N}\right)$. 
By Proposition 3.9 applied to the subspaces $\widetilde{M}_{j}$, we have $1-\frac{2 N}{N-1} \ell \leq c$. This implies $\ell^{2} \geq\left(\frac{N-1}{2 N}\right)^{2}(1-c)^{2} \geq \frac{1}{16}(1-c)^{2}$. Therefore,

$$
\begin{aligned}
\left\|T^{n}-P_{M}\right\|^{2} & =\left\|\left(Q_{N} Q_{N-1} \ldots Q_{1}\right)^{n}\right\|^{2} \leq\left\|\left(Q_{N} Q_{N-1} \ldots Q_{1}\right)\right\|^{2 n} \\
& \leq\left(1-\frac{\ell^{2}}{N^{2}}\right)^{n} \leq\left(1-\left(\frac{(1-c)^{2}}{16 N^{2}}\right)\right)^{n},
\end{aligned}
$$

which yields (i).

4C. Comparison with other estimates. Let $M_{1}, \ldots, M_{N}$ be $N$ closed subspaces of $H$, with intersection $M=M_{1} \cap \cdots \cap M_{N}$. Denote $c_{i j}=c_{0}\left(M_{i} \cap M^{\perp}, M_{j} \cap M^{\perp}\right)$ for $1 \leq i, j \leq N$.

It was proved in [13, Theorem 2.1] that

$$
\left\|\left(P_{N} \ldots P_{1}\right)^{n}-P_{M}\right\| \leq c_{1 N}^{n-1} c_{12}^{n} \ldots c_{N-1, N}^{n} .
$$

In particular, we have quick uniform convergence whenever one of the cosines $c_{i, i+1}$ of the Dixmier angles is strictly less than one.

Moreover, for any sequence $i_{1}, \ldots i_{N}$ of integers such that $\left\{i_{1}, \ldots, i_{N}\right\}=\{1, \ldots, N\}$, Theorem 4.1 shows that we have (QUC) for $\left(P_{N} \ldots P_{1}\right)^{n}$ if and only if we have (QUC) for $\left(P_{i_{N}} \ldots P_{i_{1}}\right)^{n}$. Hence, we have (QUC) for $T^{n}=\left(P_{N} \ldots P_{1}\right)^{n}$ as soon as there exist integers $i \neq j$ such that $c_{i j}=c_{0}\left(M_{i} \cap M^{\perp}, M_{j} \cap M^{\perp}\right)<1$. The following example shows that this sufficient condition for (QUC) is by far stronger than the condition $c\left(M_{1}, \ldots, M_{N}\right)<1$.

Example 4.5. Let $\left(e_{n}\right)_{n \geq 0}$ be an orthonormal basis of $H$, and let $M_{1}, M_{2}$ and $M_{3}$ be the following closed subspaces of $H: M_{1}=\overline{\operatorname{span}}\left[e_{3 n} ; n \geq 0\right], M_{2}=\overline{\operatorname{span}}\left[e_{0}, e_{3 n+1} ; n \geq 0\right]$ and $M_{3}=\overline{\operatorname{span}}\left[e_{1}, e_{3}, e_{3 n+2} ; n \geq 0\right]$. Then $M_{1} \cap M_{2}=\operatorname{span}\left[e_{0}\right], M_{2} \cap M_{3}=\operatorname{span}\left[e_{1}\right]$, $M_{1} \cap M_{3}=\operatorname{span}\left[e_{3}\right]$ and $M_{1} \cap M_{2} \cap M_{3}=\{0\}$. We obtain $c_{i j}=c_{0}\left(M_{i}, M_{j}\right)=1$ for any $i$ and $j$. But $c\left(M_{1}, M_{2}, M_{3}\right)<1$ : indeed if $x=\sum_{n \geq 0} x_{n} e_{n}$, then a straightforward computation shows that $\left\|\frac{1}{3}\left(P_{1}+P_{2}+P_{3}\right)\right\|=\frac{2}{3}$, so that $c\left(M_{1}, M_{2}, M_{3}\right)=\frac{1}{2}$.

The following proposition shows, even in a quantitative way, that the sufficient condition $c\left(M_{1} \cap \cdots \cap M_{j-1}, M_{j}\right)<1$ for each $j$, reminiscent of [31, Theorem 2.2] and [13, Theorem 2.7], implies that $c\left(M_{1}, \ldots, M_{N}\right)<1$.

Proposition 4.6. Let $N \geq 2$. Let $M_{1}, \ldots, M_{N}$ be $N$ closed subspaces of $H$ with intersection $M=M_{1} \cap \cdots \cap M_{N}$. Denote $c_{j}=c\left(M_{1} \cap \cdots \cap M_{j-1}, M_{j}\right)$ for $j$ between 2 and N. Then

$$
c\left(M_{1}, \ldots, M_{N}\right) \leq 1-\frac{1}{N-1} \prod_{j=2}^{N}\left(1-\sqrt{\frac{c_{j}+1}{2}}\right)^{2} \leq 1-\frac{1}{(N-1) 4^{N-1}} \prod_{j=2}^{N}\left(1-c_{j}\right)^{2} .
$$

In particular, $c\left(M_{1}, \ldots, M_{N}\right)<1$ if each $c_{j}, 2 \leq j \leq N$, is strictly less than 1 .

Proof. The estimates are clear if one of the $c_{j}$ 's is one. Suppose $c_{j}<1$ for every $j$. Denote $\ell_{j}=c\left(M_{1} \cap \cdots \cap M_{j-1}, M_{j}\right)>0$ for $j$ between 2 and $N$. The proof of 6 , Theorem 5.11] shows that $\ell\left(M_{1}, \ldots, M_{N}\right) \geq \ell_{2} \ell_{3} \ldots \ell_{N}$. The proof of Proposition 3.9 for $N=2$ and two given subspaces $S_{1}$ and $S_{2}$ yields

$$
1-\ell\left(S_{1}, S_{2}\right) \leq \sqrt{\kappa\left(S_{1}, S_{2}\right)}=\sqrt{\frac{c\left(S_{1}, S_{2}\right)+1}{2}} .
$$

This implies that

$$
\ell\left(S_{1}, S_{2}\right) \geq 1-\sqrt{\frac{c\left(S_{1}, S_{2}\right)+1}{2}} \geq \frac{1-c\left(S_{1}, S_{2}\right)}{4} .
$$


Using Proposition 3.9, we obtain

$$
\begin{aligned}
c\left(M_{1}, \ldots, M_{N}\right) & \leq 1-\frac{\ell\left(M_{1}, \ldots, M_{N}\right)^{2}}{N-1} \leq 1-\frac{\ell_{2}^{2} \ell_{3}^{2} \ldots \ell_{N}^{2}}{N-1} \\
& \leq 1-\frac{1}{N-1} \prod_{j=2}^{N}\left(1-\sqrt{\frac{c_{j}+1}{2}}\right)^{2} \leq 1-\frac{1}{(N-1) 4^{N-1}} \prod_{j=2}^{N}\left(1-c_{j}\right)^{2},
\end{aligned}
$$

which completes the proof.

\section{REFERENCES}

[1] I. Amemiya and T. Andô, Convergence of random products of contractions in Hilbert space, Acta Sci. Math. (Szeged) 26 (1965), 239-244. MR0187116 (32:4570)

[2] N. Aronszajn, Theory of reproducing kernels, Trans. Amer. Math. Soc. 68 (1950), $337-404$. MR0051437 (14:479c)

[3] C. Badea and Yu. I. Lyubich, Geometric, spectral and asymptotic properties of averaged products of projections in Banach spaces, Studia Math. 201 (2010), no. 1, 21-35. MR2733272

[4] C. Badea and V. Müller, On weak orbits of operators, Topology Appl. 156 (2009), 1381-1385. MR2502014 (2010b:47002)

[5] H. H. Bauschke, F. Deutsch, and H. Hundal, Characterizing arbitrarily slow convergence in the method of alternating projections, Preprint arXiv:0710.2387. Int. Trans.-Oper. Res. 16 (2009), 413-425. MR 2538248 (2010f:49030)

[6] H. H. Bauschke and J. M. Borwein, On projection algorithms for solving convex feasibility problems, SIAM Rev. 38 (1996), 367-426. MR 1409591 (98f:90045)

[7] H. H. Bauschke, J. M. Borwein, and A. S. Lewis, The method of cyclic projections for closed convex sets in Hilbert space, Recent Developments in Optimization Theory and Nonlinear Analysis (Jerusalem, 1995), Contemp. Math., vol. 204, Amer. Math. Soc., Providence, RI, 1997, pp. 1-38. MR $1442992(98 \mathrm{c}: 49069)$

[8] E. Berkson, Hermitian projections and orthogonality in Banach spaces, Proc. London Math. Soc. (3) 24 (1972), 101-118. MR0295123 (45:4191)

[9] R. E. Bruck and S. Reich, Nonexpansive projections and resolvents of accretive operators in Banach spaces, Houston J. Math. 3 (1977), 459-470. MR0470761 (57:10507)

[10] F. Deutsch, Best approximation in inner product spaces, CMS Books in Math., vol. 7, Springer, New York, 2001. MR.1823556 (2002c:41001)

[11] Rate of convergence of the method of alternating projections, Parametric Optimization and Approximation (Oberwolfach, 1983), Internat. Schriftenreihe Numer. Math., vol. 72, Birkhäuser, Basel, 1985, pp. 96-107. MR0882199 (88d:41026)

[12] _ The angle between subspaces of a Hilbert space, Approximation Theory, Wavelets and Applications (Maratea, 1994), NATO Adv. Sci. Inst. Ser. C Math. Phys. Sci., vol. 454, Kluwer Acad. Publ., Dordrecht, 1995, pp. 107-130. MR1340886 (96e:46027)

[13] F. Deutsch and H. Hundal, The rate of convergence for the method of alternating projections. II, J. Math. Anal. Appl. 205 (1997), 381-405. MR1428355 (97i:41025)

[14] J. Dye, Convergence of random products of compact contractions in Hilbert space, Integral Equations Operator Theory 12 (1989), 12-22. MR0973044(90c:47013)

[15] J. Dye, M. A. Khamsi, and S. Reich, Random products of contractions in Banach spaces, Trans. Amer. Math. Soc. 325 (1991), 87-99. MR0989572 (91h:47003)

[16] I. Halperin, The product of projection operators, Acta Sci. Math. (Szeged) 23 (1962), 96-99. MR0141978 (25:5373)

[17] S. Kayalar and H. Weinert, Error bounds for the method of alternating projections, Math. Control Signals Systems 1 (1988), 43-59. MR0923275 (89b:65137)

[18] T. Kato, Perturbation theory for linear operators, Reprint of the 1980 edition, Classics in Math., Springer, Berlin, 1995. MR1335452 (96a:47025) MR0540367 (81c:46001)

[19] B. Kirchheim, E. Kopecká, and S. Müller, Do projections stay close together? J. Math. Anal. Appl. 350 (2009), 859-871. MR2474817(2009m:51034)

[20] Y. Katznelson and L. Tzafriri, On power bounded operators, J. Funct. Anal. 68 (1986), 313-328. MR $0859138(88 \mathrm{e}: 47006)$

[21] U. Krengel, Ergodic theorems, de Gruyter Stud. Math., vol. 6, Walter de Gruyter, Berlin, 1985. MR0797411 (87i:28001)

[22] M. Lin, On the uniform ergodic theorem, Proc. Amer. Math. Soc. 43 (1974), 337-340. MR0417821 $(54: 5869)$ 
[23] J. Lindenstrauss and L. Tzafriri, Classical Banach spaces. I, II, Classics in Math., Springer, BerlinNew York, 1996. MR0500056 (58:17766)

[24] V. Müller, Power bounded operators and supercyclic vectors, Proc. Amer. Math. Soc. 131 (2003), 3807-3812. MR1999927 (2004e:47011)

[25] _ Power bounded operators and supercyclic vectors. II, Proc. Amer. Math. Soc. 133 (2005), 2997-3004. MR2159778 (2006d:47018)

[26] Spectral theory of linear operators and spectral systems in Banach algebras, Second ed., Oper. Theory Adv. Appl., vol. 139, Birkhäuser, Basel, 2007. MR2355630 (2008g:47013)

[27] J. von Neumann, On rings of operators. Reduction theory, Ann. of Math. (2) 50 (1949), 401-485. MR0029101 (10:548a)

[28] N. K. Nikol'skiı̌, Lectures on the shift operator, Nauka, Moscow, 1980; English transl., Treatise on the shift operator. Spectral function theory, Grundlehren Math. Wiss., Bd. 273, Springer-Verlag, Berlin, 1986. MR.0575166 (82i:47013); MR.0827223(87i:47042)

[29] G. Pierra, Decomposition through formalization in a product space, Math. Programming 28 (1984), 96-115. MR0727421 (85c:49044)

[30] M. Sakai, Strong convergence of infinite products of orthogonal projections in Hilbert space, Appl. Anal. 59 (1995), 109-120. MR1378029 (97a:47028)

[31] K. T. Smith, D. C. Solmon, and S. L. Wagner, Practical and mathematical aspects of the problem of reconstructing objects from radiographs, Bull. Amer. Math. Soc. 83 (1977), no. 6, 1227-1270. MR0490032(58:9394a)

[32] J. Xu and L. Zikatanov, The method of alternating projections and the method of subspace corrections in Hilbert space, J. Amer. Math. Soc. 15 (2002), 573-597. MR.1896233 (2003f:65095)

Laboratoire Paul Painlevé, Université Lille 1, CNRS UMR 8524, 59655 Villeneuve D’AscQ, FRANCE

E-mail address: badea@math.univ-lille1.fr

Laboratoire Paul Painlevé, Université Lille 1, CNRS UMR 8524, 59655 Villeneuve D’AscQ, FRANCE

E-mail address: grivaux@math.univ-lille1.fr

Institute of Mathematics AV CR, Zitna 25, 11567 Prague 1, Czech Republic

E-mail address: muller@math.cas.cz

Received 25/OCT/2009

Originally published in English 